\title{
An Analytically Tractable Model for Pricing Multiasset Options with Correlated Jump-Diffusion Equity Processes and a Two-Factor Stochastic Yield Curve
}

\author{
Tristan Guillaume \\ Laboratoire Thema, Université de Cergy-Pontoise, 33 boulevard du Port, 95011 Cergy-Pontoise Cedex, France \\ Correspondence should be addressed to Tristan Guillaume; tristan.guillaume@u-cergy.fr
}

Received 6 June 2016; Accepted 4 September 2016

Academic Editor: Peter G. L. Leach

Copyright (C) 2016 Tristan Guillaume. This is an open access article distributed under the Creative Commons Attribution License, which permits unrestricted use, distribution, and reproduction in any medium, provided the original work is properly cited.

\begin{abstract}
This paper shows how to value multiasset options analytically in a modeling framework that combines both continuous and discontinuous variations in the underlying equity or foreign exchange processes and a stochastic, two-factor yield curve. All correlations are taken into account, between the factors driving the yield curve, between fixed income and equity as asset classes, and between the individual equity assets themselves. The valuation method is applied to three of the most popular two-asset options.
\end{abstract}

\section{Introduction}

Multiasset options, that is, options whose payoff depends on more than one underlying risky asset, are actively traded in the financial markets. They are valuable to investors as tools for diversification and because they allow trading not only volatility but also correlation. They are useful to companies that need to hedge complex positions across various asset classes. For more background on these topics, the reader may refer, for example, to Bouzoubaa and Osseiran [1].

Analytical valuation formulae for multiasset options are available only in the standard Black-Scholes [2] modeling framework. The latter is notoriously based on a number of assumptions that are not empirically verified, as explained, for example, by Epps [3]. In this paper, it is shown how to price two-asset options analytically without having to rely on two of the most spurious assumptions of the Black-Scholes model, namely, the continuity of asset price paths and the nonvariability of interest rates. The former of these two assumptions is clearly invalidated by observed data in times of financial stress, especially during the so-called market crashes, in which stock prices do exhibit jumps. It results in dangerously underestimated option prices, especially for short-lived, outof-the-money contracts. As for the assumption that interest rates are constant, that is, the yield curve is flat and perfectly predictable, it becomes all the more misleading as the option expiry increases, or as the option payoff is a function of the correlation between equity and fixed income.

To overcome these two major flaws in the Black-Scholes theory, this article puts forward a model that combines a bivariate jump-diffusion equity component with a two-factor, time-dependent, stochastic yield curve. The originality of this model is to allow the simultaneous introduction of

(i) discontinuous variations in the equity prices,

(ii) stochastic evolution of the interest rate,

(iii) cross-correlation of all random factors, that is, between jumps, between the factors driving the yield curve, between fixed income and equity, and between the individual stocks themselves.

These features are introduced without having to resort to approximating numerical schemes. Complete analytical tractability is preserved, resulting in closed form formulae for option prices in terms of functions that can be numerically evaluated with the accuracy and the efficiency required for all practical purposes. To achieve analytical tractability in spite of the relatively high number of correlated random factors, the following mathematical tools are introduced: a general result on the covariance of two Ito integrals, an explicit rule for the orthogonal decomposition of a multivariate Gaussian random vector, and a computational technique allowing 
bypassing the use of a change of measure under certain conditions. The key to the derivation of the formulae is the ability to find strong solutions to the stochastic differential equations driving the variations of the underlying equity or foreign exchange assets, thus allowing obtaining the covariances between the random variables involved at the option expiry in a straightforward manner.

This article is organized as follows: Section 2 describes the model and formulates the valuation problem; Section 3 explains the method followed to solve the problem; Section 4 provides analytical formulae for three of the most widely traded multiasset options, as well as numerical results; Appendix A includes the proofs of all the lemmas introduced in Sections 2 and 3; Appendix B lists the definitions of terms used in the formulae of Section 4.

\section{Description of the Model and Formulation of the Problem}

In the sequel, $W_{t}^{(i)}$ stands for a standard Brownian motion and the constant correlation coefficient between $W_{t}^{(i)}$ and $W_{t}^{(j)}$ is denoted by $\rho_{i . j},(i, j) \in \mathbb{N}^{2}$; also, the indicator function is denoted by $\rrbracket_{(a, b)}(x)$ when it tests the inclusion of a real number $x$ within the range $(a, b)$ and by $\rrbracket_{\{\mathscr{E}\}}$ when it tests the past realization of an event defined as a subset $\mathscr{Z}$ of a given $\sigma$-algebra.

The short rate is driven by the following two-factor, timedependent, mean-reverting stochastic differential equation:

$$
d r_{t}=a\left(b(t)-r_{t}\right) d t+\sigma_{R 1} d W_{t}^{(1)}+\sigma_{R 2} d W_{t}^{(2)},
$$

where $\left(a, \sigma_{R 1}, \sigma_{R 2}\right) \in \mathbb{R}_{+}^{3}$ and $b(t)$ is a deterministic function of $t$ satisfying a linear growth condition.

This is an extended Vasicek [4] model, similar to the G2++ model of Brigo and Mercurio [5]. Compared to the original Vasicek model, a second Brownian motion has been added. This is because statistical studies of the yield curve have consistently pointed out the need to introduce at least two factors in order to reproduce the observed variability of market rates (see, e.g., [5]). In this respect, $\sigma_{R 1}$ and $\sigma_{R 2}$ are the sensitivities of the interest rate to the first and the second random factors affecting the yield curve. The mean-reversion feature has been preserved, as it is validated by empirical data, but it has been made time-dependent, so that calibration to the current market prices can be achieved by fitting an appropriate function $b(t)$.

Let $\left\{S_{t}^{(1)}, t \geq 0\right\}$ and $\left\{S_{t}^{(2)}, t \geq 0\right\}$ be the price processes of two risky assets of equity or foreign exchange type. It is assumed that they evolve in time as semimartingales with both continuous and noncontinuous sources of randomness. More precisely, the instantaneous rates of change of $S^{(1)}$ and $S^{(2)}$, under the real measure of the financial market denoted by $P$, are given, respectively, by the following stochastic differential equations (SDEs):

$$
\begin{aligned}
& \frac{d S_{t}^{(1)}}{S_{t_{-}}^{(1)}}=\left(\mu_{1}-\delta_{1}\right) d t+\sigma_{S 1} d W_{t}^{(3)}+I_{t}^{(1)} d N_{t}^{(1)} \\
& \frac{d S_{t}^{(2)}}{S_{t_{-}}^{(2)}}=\left(\mu_{2}-\delta_{2}\right) d t+\sigma_{S 2} d W_{t}^{(4)}+I_{t}^{(2)} d N_{t}^{(2)},
\end{aligned}
$$

where $\mu_{1}$ and $\mu_{2}$ are the rates of return demanded by the market to invest on $S^{(1)}$ and $S^{(2)}$, respectively, typically including a risk premium; $\delta_{1}$ and $\delta_{2}$ are constant payout rates associated with assets $S^{(1)}$ and $S^{(2)}$, respectively, for example, continuous-time equivalent dividend rates inferred from actual dividends paid at discrete dates; $\sigma_{S 1}$ is the constant diffusive volatility of $S^{(1)}$ and $\sigma_{S 2}$ is the constant diffusive volatility of $S^{(2)}$; that is, $\sigma_{S 1}$ and $\sigma_{S 2}$ are the standard deviations of the returns of $S^{(1)}$ and $S^{(2)}$, respectively, as measured with respect to the continuous part of the variations of $S^{(1)}$ and $S^{(2)} ; N_{t}^{(1)}$ and $N_{t}^{(2)}$ are two Poisson processes with intensities $\lambda_{1}$ and $\lambda_{2}$ which admit the following decomposition cases:

$$
\begin{aligned}
& N_{t}^{(1)}=Z_{t}^{(1)}+Z_{t}^{(12)}, \\
& N_{t}^{(2)}=Z_{t}^{(2)}+Z_{t}^{(12)},
\end{aligned}
$$

where $Z_{t}^{(1)}, Z_{t}^{(2)}$, and $Z_{t}^{(12)}$ are independent Poisson processes with intensities $\lambda_{1}^{\prime}, \lambda_{2}^{\prime}$, and $\lambda_{12}^{\prime}$, respectively; thus, $N_{t}^{(1)}$ and $N_{t}^{(2)}$ have positive correlation given by

$$
\rho_{1.2}^{(N)}=\frac{\lambda_{12}^{\prime}}{\sqrt{\left(\lambda_{1}^{\prime}+\lambda_{12}^{\prime}\right)\left(\lambda_{2}^{\prime}+\lambda_{12}^{\prime}\right)}},
$$

$I_{t}^{(i)}=\sum_{n} U_{n}^{(i)} \mathbb{\square}_{\left[\tau_{n-1}^{(i)}, \tau_{n}^{(i)}[\right.}(t), \forall n \in \mathbb{N}$, where $\tau_{n}^{(i)}=\inf \{t \geq$ $\left.0, N_{t}^{(i)}=n\right\}$ and $U_{n}^{(i)}$ is a sequence of independent, identically distributed random variables taking values in ] $-1,+\infty[$; in other words, $I_{t}^{(i)}$ is a right-continuous process providing the magnitude values of the jumps of asset $S_{t}^{(i)}$. Set $J_{n}^{(i)}=$ $\ln \left(1+U_{n}^{(i)}\right)$ and assume that $J_{n}^{(i)}$ is normally distributed with mean $\xi_{i}$ and variance $\varepsilon_{i}^{2}$; then,

$$
E\left[U_{n}^{(i)}\right] \triangleq \kappa_{i}=\exp \left(\xi_{i}+\frac{\varepsilon_{i}^{2}}{2}\right)-1
$$

It is assumed that all Brownian and compound Poisson processes implied by the model are defined on an adequate probability product space $\mathbb{S}$ in which the smallest $\sigma$-algebra generated by the random variables $W_{s}^{(i)}, N_{s}^{(i)}, \forall s \leq t$, and $U_{n}^{(i)} \llbracket\left\{n \leq N_{t}^{(i)}\right\}, \forall n \geq 1$, is denoted by $\mathscr{F}_{t}$.

Thus, the model used for equity is an extension of Merton [6], consisting in the introduction of a stochastic interest rate as well as of a bivariate framework allowing for multicorrelation.

As a consequence of the theory of nonarbitrage valuation of contingent claims $[7,8]$, the prices, at the current time denoted by $t_{0}$, of European call or put options written on $S^{(1)}$ and $S^{(2)}$ with expiry $T$, can be expressed as linear combinations of conditional expectations of two kinds:

$$
\begin{gathered}
E_{\mathrm{Q}}\left[\exp \left(-\int_{0}^{T} r_{t} d t\right) f\left(S_{T}^{(1)}, S_{T}^{(2)}\right) \llbracket\{\mathscr{Z}\} \mid \mathscr{F}_{0}\right] \\
E_{\mathrm{Q}}\left[\exp \left(-\int_{0}^{T} r_{t} d t\right) \square\{\mathscr{Z}\} \mid \mathscr{F}_{0}\right] .
\end{gathered}
$$


In (7) and (8), $\mathscr{Z}$ represents an event involving $S_{T}^{(1)}$ and/or $S_{T}^{(2)}$ that has to occur for the option to have strictly positive value at expiry $T$, while $f$ is a function implied by the specific payoff under consideration, and $Q$ is an equivalent martingale measure. Let us first give examples of $\mathscr{Z}$ and $f$, assuming $Q$ is known. To begin with, consider a put option on the minimum of two assets. This popular contract pays out the difference, if positive, between a prespecified strike price $K$ and the minimum between two equity asset prices at expiry $T$. Its Black-Scholes price is given by Stulz [9]. In our model, the noarbitrage value of this contingent claim can be decomposed as follows:

$$
\begin{gathered}
K E_{\mathrm{Q}}\left[\exp \left(-\int_{0}^{T} r_{t} d t\right) I_{1} \mid \mathscr{F}_{0}\right] \\
-E_{\mathrm{Q}}\left[\exp \left(-\int_{0}^{T} r_{t} d t\right) \alpha_{1} S_{T}^{(1)} I_{1} \mid \mathscr{F}_{0}\right] \\
+K E_{\mathrm{Q}}\left[\exp \left(-\int_{0}^{T} r_{t} d t\right) I_{2} \mid \mathscr{F}_{0}\right] \\
-E_{\mathrm{Q}}\left[\exp \left(-\int_{0}^{T} r_{t} d t\right) \alpha_{2} S_{T}^{(2)} I_{2} \mid \mathscr{F}_{0}\right],
\end{gathered}
$$

where $\alpha_{1} \in(0,1)$ and $\alpha_{2} \in(0,1)$ are predefined weights attached to $S^{(1)}$ and $S^{(2)}$, respectively, and $I_{1}$ and $I_{2}$ are indicator functions defined by

$$
\begin{aligned}
& I_{1}=\rrbracket_{\left\{\alpha_{1} S_{T}^{(1)}<K, \alpha_{1} S_{T}^{(1)}<\alpha_{2} S_{T}^{(2)}\right\}}
\end{aligned}
$$

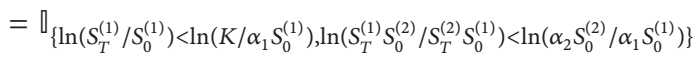

$$
\begin{aligned}
& I_{2}=\rrbracket_{\left\{\alpha_{2} S_{T}^{(2)}<K, \alpha_{2} S_{T}^{(2)}<\alpha_{1} S_{T}^{(1)}\right\}}
\end{aligned}
$$

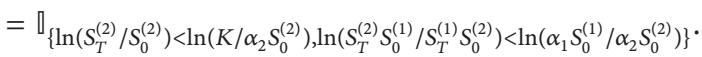

Our second example of actively traded instrument is the (zero-strike) spread option, which yields the difference between the higher and the lower of two asset prices at expiry $T$. Its Black-Scholes price was first obtained by Margrabe [10]. In our model, the no-arbitrage value of this contract can be written as

$$
\begin{array}{r}
E_{\mathrm{Q}}\left[\exp \left(-\int_{0}^{T} r_{t} d t\right) \alpha_{1} S_{T}^{(1)} I_{3} \mid \mathscr{F}_{0}\right] \\
-E_{\mathrm{Q}}\left[\exp \left(-\int_{0}^{T} r_{t} d t\right) \alpha_{2} S_{T}^{(2)} I_{3} \mid \mathscr{F}_{0}\right] \\
+E_{\mathrm{Q}}\left[\exp \left(-\int_{0}^{T} r_{t} d t\right) \alpha_{2} S_{T}^{(2)} I_{4} \mid \mathscr{F}_{0}\right] \\
-E_{\mathrm{Q}}\left[\exp \left(-\int_{0}^{T} r_{t} d t\right) \alpha_{1} S_{T}^{(1)} I_{4} \mid \mathscr{F}_{0}\right],
\end{array}
$$

where $I_{3}$ and $I_{4}$ are indicator functions defined by

$$
\begin{aligned}
& I_{3}=\mathbb{\square}_{\left\{\alpha_{1} S_{T}^{(1)}>\alpha_{2} S_{T}^{(2)}\right\}}=\mathbb{Z}_{\left\{\ln \left(S_{T}^{(1)} S_{0}^{(2)} / S_{T}^{(2)} S_{0}^{(1)}\right)>\ln \left(\alpha_{2} S_{0}^{(2)} / \alpha_{1} S_{0}^{(1)}\right)\right\}} \\
& I_{4}=\mathbb{q}_{\left\{\alpha_{2} S_{T}^{(2)}>\alpha_{1} S_{T}^{(1)}\right\}}=\mathbb{\square}_{\left\{\ln \left(S_{T}^{(2)} S_{0}^{(1)} / S_{T}^{(1)} S_{0}^{(2)}\right)>\ln \left(\alpha_{1} S_{0}^{(1)} / \alpha_{2} S_{0}^{(2)}\right)\right\}} .
\end{aligned}
$$

Our third example is a call option on a product, which pays out the difference, if it is positive, between the product of two asset prices, $S^{(1)}$ and $S^{(2)}$, and a prespecified strike price $K$ at expiry $T$. Usually, in a product option, $S^{(1)}$ is a foreign exchange rate, while $S^{(2)}$ is a foreign currency denominated equity price, and the contract is often called a quanto option. The Black-Scholes value of the latter can be found in Zhang [11]. In our model, the no-arbitrage value of this contract can be written as

$$
\begin{gathered}
E_{\mathrm{Q}}\left[\exp \left(-\int_{0}^{T} r_{t} d t\right) S_{T}^{(1)} S_{T}^{(2)} I_{5} \mid \mathscr{F}_{0}\right] \\
-K E_{\mathrm{Q}}\left[\exp \left(-\int_{0}^{T} r_{t} d t\right) I_{5} \mid \mathscr{F}_{0}\right],
\end{gathered}
$$

where $I_{5}$ is an indicator function defined by

$$
I_{5}=\mathbb{q}_{\left\{S_{T}^{(1)} S_{T}^{(2)}>K\right\}}=\mathbb{\square}_{\left\{\ln \left(S_{T}^{(1)} S_{T}^{(2)} / S_{0}^{(1)} S_{0}^{(2)}\right)>\ln \left(K / S_{0}^{(1)} S_{0}^{(2)}\right)\right\}} \cdot
$$

Let us now move on to the question of the pricing measure $Q$. As a result of the theory of no-arbitrage valuation, $Q$ must be the equivalent martingale measure under which the numeraire is the so-called money market account $\beta_{t}$, defined by $\beta_{t}=\left\{\exp \left(\int_{0}^{t} r_{s} d s\right), t \geq 0\right\}$. Let us recall that a numeraire is a financial asset whose price can be modeled by a strictly positive stochastic process, while a probability measure $P$ is said to be martingale equivalent for a given numeraire $N$ if the price of any asset divided by $N$ is a martingale under $P$ [12]. The following lemma is introduced in order to determine the dynamics of $S^{(1)}$ and $S^{(2)}$ under $Q$.

Lemma 1. Using the previously defined notations, let $\left\{S_{t}, t \geq\right.$ $0\}$ be a process driven by $d S_{t} / S_{t_{-}}=\mu_{t} d t+\sigma d W_{t}+I_{t} d N_{t}$, where $\mu_{t}$ is a continuous $\mathscr{F}_{t}$-adapted process verifying the usual assumptions for the latter stochastic differential equation to be well defined. Then, denoting by $\lambda$ the intensity of the Poisson process $\left\{N_{t}, t \geq 0\right\}$, a necessary condition for the discounted asset price $\widetilde{S}_{T} \triangleq \exp \left(-\int_{0}^{T} r_{t} d t\right) S_{T}$ to be a martingale is to have $\mu_{t}=r_{t}-\lambda \kappa$

\section{Proof of Lemma 1. See Appendix A.}

Hence, the instantaneous rates of change of $S^{(1)}$ and $S^{(2)}$ under $Q$ are driven, respectively, by the following equations:

$$
\begin{aligned}
& \frac{d S_{t}^{(1)}}{S_{t_{-}}^{(1)}}=\left(r_{t}-\delta_{1}-\lambda_{1} \kappa_{1}\right) d t+\sigma_{S 1} d W_{t}^{(3)}+I_{t}^{(1)} d N_{t}^{(1)} \\
& \frac{d S_{t}^{(2)}}{S_{t_{-}}^{(2)}}=\left(r_{t}-\delta_{2}-\lambda_{2} \kappa_{2}\right) d t+\sigma_{S 2} d W_{t}^{(4)}+I_{t}^{(2)} d N_{t}^{(2)} .
\end{aligned}
$$

It is emphasized that the condition given in Lemma 1 is necessary but not sufficient. Actually, the introduction of jumps makes the market incomplete; that is, perfect hedging is not possible. Merton [6] argues that jump risk is diversifiable and therefore not rewardable with excess return. However, that argument is debatable because of the existence 
of industrywide and countrywide shocks. In this respect, two kinds of jumps can be considered in our model. The "idiosyncratic" ones refer to shocks that specifically affect a single company instead of the market or a whole economic sector. This is the kind of jumps that can be diversified. On the other hand, "systemic" jumps affect all stocks, as can be observed during market crashes. The nature of risk can be defined by adjusting the parameter $\lambda_{12}^{\prime}$ : the lower this parameter is, the more the jump risk becomes idiosyncratic. The magnitude of the parameters $\lambda_{1}^{\prime}$ and $\lambda_{2}^{\prime}$ reflects the degree of diversification: in a perfectly diversified portfolio, these parameters should tend to zero, so that the compound Poisson processes driving the two stocks have the same market-driven intensity $\lambda_{12}^{\prime}$.

\section{Analytical Method of Valuation}

In order to price options on $S_{T}^{(1)}$ and $S_{T}^{(2)}$, SDEs (23) and (24) must be solved, conditional on $N_{T}^{(1)}$ and $N_{T}^{(2)}$. An application of Ito's lemma to $\ln \left(S_{t}^{(1)}\right)$ and to $\ln \left(S_{t}^{(2)}\right)$ yields the following solutions to (23) and (24) at time $T$ :

$$
\begin{aligned}
& S_{T}^{(1)} \mid N_{T}^{(1)}=S_{0}^{(1)} \exp \left(\int_{0}^{T} r_{t} d s\right. \\
& \left.-\left(\delta_{1}+\lambda_{1} \kappa_{1}+\frac{\sigma_{S 1}^{2}}{2}\right) T+\sigma_{S 1} W_{T}^{(3)}+\sum_{i=1}^{N_{T}^{(1)}} J_{i}^{(1)}\right) \\
& S_{T}^{(2)} \mid N_{T}^{(2)}=S_{0}^{(2)} \exp \left(\int_{0}^{T} r_{t} d t\right. \\
& \left.-\left(\delta_{2}+\lambda_{2} \kappa_{2}+\frac{\sigma_{S 2}^{2}}{2}\right) T+\sigma_{S 2} W_{T}^{(4)}+\sum_{i=1}^{N_{T}^{(2)}} J_{i}^{(2)}\right),
\end{aligned}
$$

where $\sum_{i=1}^{N_{T}^{(1)}} J_{i}^{(1)} \mid N_{T}^{(1)}$ and $\sum_{i=1}^{N_{T}^{(2)}} J_{i}^{(2)} \mid N_{T}^{(2)}$ are normally distributed with $E\left[\sum_{i=1}^{N_{T}^{(k)}} J_{i}^{(k)} \mid N_{T}^{(k)}\right]=N_{T}^{(k)} \xi_{k}$ and $\operatorname{var}\left[\sum_{i=1}^{N_{T}^{(k)}} J_{i}^{(k)} \mid\right.$ $\left.N_{T}^{(k)}\right]=N_{T}^{(k)} \varepsilon_{k}^{2}, \forall k \in\{1,2\}$.

Solutions (25) are not explicit enough to compute option values. They are only representations of the solutions to (23) and (24) in terms of the integrated interest rate. They do not give the correlation structure between fixed income and equity, that is, the dependence of assets $S^{(1)}$ and $S^{(2)}$ on the Brownian motions $W^{(1)}$ and $W^{(2)}$. Consequently, neither do they give the correlation structure between the continuous parts of the returns on $S^{(1)}$ and $S^{(2)}$, conditional on the correlation between fixed income and equity, that is, the correlation between $W^{(3)}$ and $W^{(4)}$ conditional on $W^{(1)}$ and $W^{(2)}$. First, to determine explicitly the correlation between fixed income and equity, let us focus on the time integral of $r_{t}$ on $[0, T]$, that is, on $\int_{0}^{T} r_{t} d t$. By taking the stochastic differential of $\exp (a t) r_{t}$ and integrating it on $[0, t]$, (1) yields

$$
\begin{aligned}
r_{t}= & r_{0} e^{-a t}+a \int_{0}^{t} e^{-a(t-s)} b(s) d s \\
& +\sigma_{R 1} \int_{0}^{t} e^{-a(t-s)} d W_{s}^{(1)}+\sigma_{R 2} \int_{0}^{t} e^{-a(t-s)} d W_{s}^{(2)} .
\end{aligned}
$$

The integral $\int_{0}^{T} r_{t} d t$ is normally distributed since $r_{t}$ is a Gaussian process. Fubini's theorem yields $E\left[\int_{0}^{T} r_{t} d t\right]=\bar{\mu}_{r}$, as given in the Appendix. The variance of $\int_{0}^{T} r_{t} d t$ can be written as $\operatorname{var}\left[\int_{0}^{T} r_{t} d t\right]=\int_{0}^{T} \int_{0}^{t} \operatorname{cov}\left[r_{t}, r_{s}\right] d s d t$. To tackle this integral, the following lemma is introduced.

Lemma 2. Let $\left\{W_{s}^{(1)}, s \geq 0\right\}$ and $\left\{W_{s}^{(2)}, s \geq 0\right\}$ be two standard Brownian motions with constant correlation coefficient $\rho$. The natural filtration generated by $\left(W_{s}^{(1)}, W_{s}^{(2)}\right)$ is denoted by $\mathscr{F}_{s}$. Let $\left\{Y_{s}^{(1)}, s \geq 0\right\}$ and $\left\{Y_{s}^{(2)}, s \geq 0\right\}$ be two rightcontinuous, $\mathscr{F}_{s}$-adapted processes such that $E\left[\int_{0}^{T}\left(Y_{s}^{(1)}\right)^{2} d s\right]<$ $\infty$ and $E\left[\int_{0}^{T}\left(Y_{s}^{(2)}\right)^{2} d s\right]<\infty$. Then, $\forall 0 \leq t \leq T$, one has

$$
\begin{aligned}
\operatorname{cov} & {\left[\int_{0}^{t} Y_{s}^{(1)} d W_{s}^{(1)}, \int_{0}^{T} Y_{s}^{(2)} d W_{s}^{(2)}\right] } \\
& =\rho \int_{0}^{t} E\left[Y_{s}^{(1)} Y_{s}^{(2)}\right] d s .
\end{aligned}
$$

Proof of Lemma 2. See Appendix A.

Lemma 2 yields the covariance function of $\{r(t), t \geq 0\}$ :

$$
\begin{aligned}
\operatorname{cov} & {\left[r_{t}, r_{s}\right] } \\
& =e^{-a(s+t)}\left(\frac{e^{2 a s}-1}{2 a}\right)\left(\sigma_{R 1}^{2}+\sigma_{R 2}^{2}+2 \rho_{1.2} \sigma_{R 1} \sigma_{R 2}\right),
\end{aligned}
$$

so that the variance of the time integral of $\{r(t), t \geq 0\}$ is equal to

$$
\begin{gathered}
\operatorname{var}\left[\int_{0}^{T} r_{t} d t\right]=\frac{1}{a^{2}}\left(\sigma_{R 1}^{2}+\sigma_{R 2}^{2}+2 \rho_{1.2} \sigma_{R 1} \sigma_{R 2}\right) \\
\cdot\left(T+\frac{2\left(e^{-a T}-1\right)}{a}+\frac{1-e^{-2 a T}}{2 a}\right) .
\end{gathered}
$$

Thus, for fixed $T \geq 0$, the following equality holds in law:

$$
\begin{aligned}
& \int_{0}^{T} r_{t} d t \\
& \quad=\bar{\mu}_{r}+\bar{\sigma}_{r}\left(W_{T}^{(1)}\left(\sigma_{R 1}+\sigma_{R 2} \rho_{1.2}\right)+\sigma_{R 2} \sigma_{2 \mid 1} \bar{W}_{T}^{(2)}\right),
\end{aligned}
$$

where

$$
\bar{\sigma}_{r}=\frac{1}{a \sqrt{T}} \sqrt{T-\frac{2}{a}\left(1-e^{-a T}\right)+\frac{1}{2 a}\left(1-e^{-2 a T}\right)} .
$$


The next step is to determine explicitly the correlation structure between the continuous parts of the returns on $S^{(1)}$ and $S^{(2)}$, conditional on the correlation between fixed income and equity. This can be achieved by using the following lemma.

Lemma 3. At any given time $t \geq 0, W_{t}^{(2)}, W_{t}^{(3)}$, and $W_{t}^{(4)}$ can be written as follows:

$$
\begin{aligned}
W_{t}^{(2)}= & \rho_{1.2} W_{t}^{(1)}+\sigma_{2 \mid 1} \bar{W}_{t}^{(2)}(t) \\
W_{t}^{(3)}= & \rho_{1.3} W_{t}^{(1)}+\rho_{2.3 \mid 1} \bar{W}_{t}^{(2)}+\sigma_{3 \mid 1.2} \bar{W}_{t}^{(3)} \\
W_{t}^{(4)}= & \rho_{1.4} W_{t}^{(1)}+\rho_{2.4 \mid 1} \bar{W}_{t}^{(2)}+\rho_{3.4 \mid 1.2} \bar{W}_{t}^{(3)} \\
& +\sigma_{4 \mid 1.2 .3} \bar{W}_{t}^{(4)},
\end{aligned}
$$

where $\left\{\bar{W}_{t}^{(2)}, t \geq 0\right\},\left\{\bar{W}_{t}^{(3)}, t \geq 0\right\}$, and $\left\{\bar{W}_{t}^{(4)}, t \geq 0\right\}$ are standard Brownian motions independent both of one another and of $\left\{W_{t}^{(1)}, t \geq 0\right\}$ and where the following definitions hold:

$$
\begin{aligned}
\sigma_{2 \mid 1} & =\sqrt{1-\rho_{1.2}^{2}} \\
\rho_{2.3 \mid 1} & =\frac{\rho_{2.3}-\rho_{1.2} \rho_{1.3}}{\sigma_{2 \mid 1}} \\
\sigma_{3 \mid 1.2} & =\sqrt{1-\rho_{1.3}^{2}-\rho_{2.3 \mid 1}^{2}} \\
\rho_{2.4 \mid 1} & =\frac{\rho_{2.4}-\rho_{1.2} \rho_{1.4}}{\sigma_{2 \mid 1}} \\
\rho_{3.4 \mid 1.2} & =\frac{\rho_{3.4}-\rho_{1.3} \rho_{1.4}-\rho_{2.3 \mid 1} \rho_{2.4 \mid 1}}{\sigma_{3 \mid 1.2}} \\
\sigma_{4 \mid 1.2 .3} & =\sqrt{1-\rho_{1.4}^{2}-\rho_{2.4 \mid 1}^{2}-\rho_{3.4 \mid 1.2}^{2}} .
\end{aligned}
$$

Proof of Lemma 3. See Appendix A.

Thus, a combination of Lemmas 1, 2, and 3 yields, under the pricing measure $Q$, the following strong solutions to SDEs (23) and (24), conditional on $N_{T}^{(1)}$ and $N_{T}^{(2)}$, in which all the correlations are explicit:

$$
\begin{aligned}
\ln \frac{S_{T}^{(1)}}{S_{0}^{(1)}}= & \bar{\mu}_{r}-\left(\delta_{1}+\lambda_{1} \kappa_{1}+\frac{\sigma_{S 1}^{2}}{2}\right) T \\
& +W_{T}^{(1)}\left(\bar{\sigma}_{r}\left(\sigma_{R 1}+\sigma_{R 2} \rho_{1.2}\right)+\sigma_{S 1} \rho_{1.3}\right) \\
& +\bar{W}_{T}^{(2)}\left(\bar{\sigma}_{r} \sigma_{R 2} \sigma_{2 \mid 1}+\sigma_{S 1} \rho_{2.3 \mid 1}\right) \\
& +\bar{W}_{T}^{(3)} \sigma_{S 1} \sigma_{3 \mid 1.2}+\sum_{i=1}^{N_{T}^{(1)}} J_{i}^{(1)}
\end{aligned}
$$

$$
\begin{aligned}
\ln \frac{S_{T}^{(2)}}{S_{0}^{(2)}}= & \bar{\mu}_{r}-\left(\delta_{2}+\lambda_{2} \kappa_{2}+\frac{\sigma_{S 2}^{2}}{2}\right) T \\
& +W_{T}^{(1)}\left(\bar{\sigma}_{r}\left(\sigma_{R 1}+\sigma_{R 2} \rho_{1.2}\right)+\sigma_{S 2} \rho_{1.4}\right) \\
& +\bar{W}_{T}^{(2)}\left(\bar{\sigma}_{r} \sigma_{R 2} \sigma_{2 \mid 1}+\sigma_{S 2} \rho_{2.4 \mid 1}\right) \\
& +\bar{W}_{T}^{(3)} \sigma_{S 2} \rho_{3.4 \mid 1.2}+\bar{W}_{T}^{(4)} \sigma_{S 2} \sigma_{4 \mid 1.2 .3} \\
& +\sum_{i=1}^{N_{T}^{(2)}} J_{i}^{(2)} .
\end{aligned}
$$

Since all the random variables summed in (36) are normally distributed and pairwise independent, the conditional distributions of $\ln \left(S_{T}^{(1)} / S_{0}^{(1)}\right)$ and $\ln \left(S_{T}^{(2)} / S_{0}^{(2)}\right)$ under $Q$ are Gaussian, and so are those of $\ln \left(S_{T}^{(1)} S_{0}^{(2)} / S_{T}^{(2)} S_{0}^{(1)}\right), \ln \left(S_{T}^{(2)} S_{0}^{(1)} / S_{T}^{(1)} S_{0}^{(2)}\right)$, and $\ln \left(S_{T}^{(1)} S_{T}^{(2)} / S_{0}^{(1)} S_{0}^{(2)}\right)$. The parameters of these distributions follow easily from stability under addition and are listed in Appendix B.

We can now turn to the computation of (7) and (8). A classical way of turning an expectation involving an indicator function into a probability, that is, of getting rid of any random function multiplying the indicator function inside the expectation operator, is to switch to a new measure. In this respect, the following lemma is instrumental in computing the expectations of the second kind in (8).

Lemma 4. Denoting by $\mathscr{Z}$ a subset of the $\sigma$-algebra generated by the random variables defined on the space $\mathbb{S}$, one has

$$
\begin{gathered}
E_{\mathrm{Q}}\left[\exp \left(-\int_{0}^{T} r_{t} d t\right) \square\{\mathscr{E}\} \mid \mathscr{F}_{0}\right] \\
=B(0, T) E_{P_{B_{T}}}\left[\square\{\mathscr{E}\} \mid \mathscr{F}_{0}\right],
\end{gathered}
$$

where

$$
\begin{aligned}
& \begin{aligned}
& B(t, T)=\exp \left(-A(t, T) r_{t}+C(t, T)\right), \quad 0 \leq t \leq T \\
& A(t, T)=\frac{1}{a}\left(1-e^{-a(T-t)}\right) \\
& C(t, T)=-a \int_{t}^{T}\left(\int_{t}^{u} e^{-a(u-s)} b(s) d s\right) d u \\
&+\frac{1}{a^{2}}\left(\sigma_{R 1}^{2}+\sigma_{R 2}^{2}+2 \rho_{1.2} \sigma_{R 1} \sigma_{R 2}\right) \\
& \cdot\left(T-t+\frac{2\left(e^{-a(T-t)}-1\right)}{a}+\frac{1-e^{-2 a(T-t)}}{2 a}\right)
\end{aligned} \\
& \text { and } P_{B_{T}} \text { is a measure equivalent to Q. } \\
& \text { Proof of Lemma 4. See Appendix A. }
\end{aligned}
$$


As a consequence of Lemma 4, we obtain

$$
\begin{aligned}
W_{T}^{(1)} & =Z_{T}^{(1)}-\left(\sigma_{R 1}+\sigma_{R 2} \rho_{1.2}\right) \int_{0}^{T} A(s, T) d s \\
& =Z_{T}^{(1)}+\frac{\left(\sigma_{R 1}+\sigma_{R 2} \rho_{1.2}\right)\left(1-e^{-a T}-T\right)}{a^{2}} \\
\bar{W}_{T}^{(2)} & =\bar{Z}_{T}^{(2)}-\sigma_{R 2} \sigma_{2 \mid 1} \int_{0}^{T} A(s, T) d s \\
& =\bar{Z}_{T}^{(2)}+\frac{\sigma_{R 2} \sigma_{2 \mid 1}\left(1-e^{-a T}-T\right)}{a^{2}},
\end{aligned}
$$

where $Z^{(1)}$ and $\bar{Z}^{(2)}$ are independent standard Brownian motions under $P_{B_{T}}$.

Given $N_{T}^{(1)}$ and $N_{T}^{(2)}$, one can then easily find strong solutions for $S_{T}^{(1)}$ and $S_{T}^{(2)}$ under $P_{B_{T}}$ by substituting (39) into (36).

To compute the expectations of the first kind in (7), one can draw on the joint normality of the involved random variables and use the following lemma.

Lemma 5. Let $X \sim N\left(\mu_{X}, \sigma_{X}^{2}\right), Y \sim N\left(\mu_{Y}, \sigma_{Y}^{2}\right)$, and $Z \sim$ $N\left(\mu_{Z}, \sigma_{Z}^{2}\right)$ be three normal random variables with constant correlation coefficients denoted by $\rho_{X . Y}, \rho_{X . Z}$, and $\rho_{Y . Z}$. Let a and $b$ be two real numbers. Then,

$$
\begin{aligned}
& E[\exp (X) \square\{Y \leq a, Z \leq b\}]=\exp \left(\mu_{X}+\frac{\sigma_{X}^{2}}{2}\right) \\
& \cdot N_{2}\left[\frac{a-\mu_{Y}-\rho_{X . Y} \sigma_{X} \sigma_{Y}}{\sigma_{Y}}, \frac{b-\mu_{Z}-\rho_{X . Z} \sigma_{X} \sigma_{Z}}{\sigma_{Z}} ;\right. \\
& \left.\rho_{Y . Z}\right] \\
& E[\exp (X) \square\{Y \leq a\}]=\exp \left(\mu_{X}+\frac{\sigma_{X}^{2}}{2}\right) \\
& \cdot N\left[\frac{a-\mu_{Y}-\rho_{X . Y} \sigma_{X} \sigma_{Y}}{\sigma_{Y}}\right],
\end{aligned}
$$

where $N[a]$ is the univariate standard normal cumulative distribution function with upper bound $a \in \mathbb{R}$ and $N_{2}[a, b ; \rho]$ is the bivariate standard normal cumulative distribution function with upper bounds $a \in \mathbb{R}$ and $b \in \mathbb{R}$, and correlation coefficient $\rho \in]-1,1[$.

\section{Proof of Lemma 5. See Appendix A.}

Applying the five previous lemmas, it only remains to sum over the joint distribution of $\left(N_{T}^{(1)}, N_{T}^{(2)}\right)$, which is the product of the distributions of $Z_{T}^{(1)}, Z_{T}^{(2)}$, and $Z_{T}^{(12)}$, to obtain closed form option pricing formulae, as the next section shows.

\section{Formulae and Numerical Results}

Consider first the value of a put option on the minimum of two assets with strike price $K$, as described in Section 2. The pairs of random variables $\left(\ln \left(S_{T}^{(1)} S_{0}^{(2)} / S_{T}^{(2)} S_{0}^{(1)}\right), \ln \left(S_{T}^{(1)} / S_{0}^{(1)}\right)\right)$ and $\left(\ln \left(S_{T}^{(2)} S_{0}^{(1)} / S_{T}^{(1)} S_{0}^{(2)}\right), \ln \left(S_{T}^{(2)} / S_{0}^{(2)}\right)\right)$ are both bivariate normal and the implied pairwise conditional covariances, given by $\Phi_{3}$ and $\Phi_{6}$ in Appendix B, are easily derived using (36). To compute conditional expectations (9) and (11), Lemma 4 is applied to obtain $E_{P_{B_{T}}}\left[\begin{array}{lll}I_{1} & \mid & \mathscr{F}_{0}\end{array}\right]$ and $E_{P_{B_{T}}}\left[\begin{array}{lll}I_{2} & \mid \mathscr{F}_{0}\end{array}\right]$, respectively.

To compute conditional expectation (10), Lemma 5 is applied with

$$
\begin{aligned}
& X=\left(-\int_{0}^{T} r_{t} d t\right)+\ln \left(\frac{S_{T}^{(1)}}{S_{0}^{(1)}}\right), \\
& Y=\ln \left(\frac{S_{T}^{(1)}}{S_{0}^{(1)}}\right), \\
& Z=\ln \left(\frac{S_{T}^{(1)} S_{0}^{(2)}}{S_{T}^{(2)} S_{0}^{(1)}}\right) .
\end{aligned}
$$

To compute conditional expectation (12), Lemma 5 is applied with

$$
\begin{aligned}
& X=\left(-\int_{0}^{T} r_{t} d t\right)+\ln \left(\frac{S_{T}^{(2)}}{S_{0}^{(2)}}\right), \\
& Y=\ln \left(\frac{S_{T}^{(2)}}{S_{0}^{(2)}}\right), \\
& Z=\ln \left(\frac{S_{T}^{(2)} S_{0}^{(1)}}{S_{T}^{(1)} S_{0}^{(2)}}\right) .
\end{aligned}
$$

Generalizing to a put or a call option on the minimum or on the maximum of two assets, the following formula can be obtained.

Formula 1. Let $V\left(\alpha_{1}, \alpha_{2} ; S_{0}^{(1)}, S_{0}^{(2)} ; K ; T\right)$ denote the noarbitrage value at time $t_{0}=0$ of a put or a call on the minimum or on the maximum at expiry $T$ of two weighted assets $\alpha_{1} S^{(1)}$ and $\alpha_{2} S^{(2)}$ with strike price $K$. Within the modeling framework defined in Section 2, we have

$$
\begin{aligned}
V & \left(\alpha_{1}, \alpha_{2} ; S_{0}^{(1)}, S_{0}^{(2)} ; K ; T\right) \\
& =\sum_{n_{1}=0}^{\infty} \sum_{n_{2}=0}^{\infty} \sum_{n_{12}=0}^{\infty} e^{-\lambda_{1}^{\prime} T-\lambda_{2}^{\prime} T-\lambda_{12}^{\prime} T} \\
& . \frac{\left(\lambda_{1}^{\prime} T\right)^{n_{1}}\left(\lambda_{2}^{\prime} T\right)^{n_{2}}\left(\lambda_{12}^{\prime} T\right)^{n_{12}}}{n_{1} ! n_{2} ! n_{12} !}\left\{\Upsilon \alpha _ { 1 } S _ { 0 } ^ { ( 1 ) } \operatorname { e x p } \left(\nu_{1}\right.\right. \\
+ & \left.\frac{\gamma_{1}^{2}}{2}\right) N_{2}\left[\Upsilon \frac{\ln \left(\alpha_{1} S_{0}^{(1)} / K\right)+\nu_{2}+\Phi_{1}}{\gamma_{2}}, \theta\right. \\
& \left.\frac{\vartheta(1,2)+\nu_{3}+\Phi_{2}}{\gamma_{3}} \Upsilon \Upsilon \frac{\Phi_{3}}{\gamma_{2} \gamma_{3}}\right]+\Upsilon \alpha_{2} S_{0}^{(2)} \exp \left(\nu_{4}\right. \\
+ & \left.\frac{\gamma_{4}^{2}}{2}\right) N_{2}\left[\Upsilon \frac{\ln \left(\alpha_{2} S_{0}^{(2)} / K\right)+\nu_{5}+\Phi_{4}}{\gamma_{5}}, \theta\right.
\end{aligned}
$$


TABLE 1: Values of put options on the minimum of two assets ${ }^{\mathrm{a}}$.

\begin{tabular}{|c|c|c|c|c|}
\hline & $\begin{array}{l}\text { 3-month expiry put } \\
\text { option on minimum } \\
\qquad K=100\end{array}$ & $\begin{array}{l}\text { 3-month expiry put } \\
\text { option on minimum } \\
K=90\end{array}$ & $\begin{array}{c}\text { 1-year expiry put option } \\
\text { on minimum } \\
K=100\end{array}$ & $\begin{array}{c}\text { 1-year expiry put option } \\
\text { on minimum } \\
K=90\end{array}$ \\
\hline $\begin{array}{l}\text { Model 1: } \\
\text { Black-Scholes }\end{array}$ & 9.304886727 & 3.630237975 & 17.0518359 & 10.66664224 \\
\hline $\begin{array}{l}\text { Model 2: no jumps, } \\
\text { stochastic interest rate }\end{array}$ & 9.62905761 & 2.87924378 & 19.1872084 & 11.5275656 \\
\hline $\begin{array}{l}\text { Model 3: low intensity } \\
\text { jumps, constant } \\
\text { interest rate }\end{array}$ & 11.2923396 & 4.64155701 & 21.0275563 & 13.5964336 \\
\hline $\begin{array}{l}\text { Model } 4 \text { : low intensity } \\
\text { jumps, stochastic } \\
\text { interest rate }\end{array}$ & 10.4286542 & 3.61484021 & 20.6860149 & 12.9592418 \\
\hline $\begin{array}{l}\text { Model 5: high } \\
\text { intensity jumps, } \\
\text { constant interest rate }\end{array}$ & 11.8892729 & 5.15613322 & 22.3691619 & 14.8593002 \\
\hline $\begin{array}{l}\text { Model 6: high } \\
\text { intensity jumps, } \\
\text { stochastic interest rate }\end{array}$ & 11.0618998 & 4.16206372 & 22.0902324 & 14.307958 \\
\hline \multicolumn{5}{|c|}{$\begin{array}{l}\text { all values other than Black-Scholes were computed by means of Formula } 1 \text { with the following inputs: } \alpha_{1}=\alpha_{2}=1 S_{0}^{(1)}=S_{0}^{(2)}=100, \sigma_{S 1}=35 \%, \sigma_{S 2}=28 \% \\
\delta_{1}=\delta_{2}=0, \rho_{1.2}=0.3, \rho_{1.3}=-0.25, \rho_{1.4}=-0.28, \rho_{2.3}=-0.22, \rho_{2.4}=-0.26, \rho_{3.4}=-0.36, \xi_{1}=\xi_{2}=0, \varepsilon_{1}=0.2 \text {, and } \varepsilon_{2}=0.3 \text {. } \\
\text { The "constant interest rate" setting is defined by taking an interest rate equal to } 3 \% \text {. } \\
\text { The "stochastic interest rate" setting is defined by taking } r_{0}=0.03, b(t)=0.03, a=0.6, \sigma_{R 1}=0.08 \text {, and } \sigma_{R 2}=0.12 \text {. } \\
\text { The "low intensity jumps" setting is defined by taking } \lambda_{1}^{\prime}=0.2, \lambda_{2}^{\prime}=0.3 \text {, and } \lambda_{12}^{\prime}=0 . \\
\text { The "high intensity jumps" setting is defined by taking } \lambda_{1}^{\prime}=0.4, \lambda_{2}^{\prime}=0.6 \text {, and } \lambda_{12}^{\prime}=0 .\end{array}$} \\
\hline
\end{tabular}

$$
\begin{aligned}
& \left.\cdot \frac{\vartheta(2,1)-\nu_{3}+\Phi_{5}}{\gamma_{3}} ; \Upsilon \theta \frac{\Phi_{6}}{\gamma_{5} \gamma_{3}}\right]-\Upsilon B(0, T) \\
& \cdot K\left(N _ { 2 } \left[\Upsilon \frac{\ln \left(\alpha_{1} S_{0}^{(1)} / K\right)+\nu_{6}}{\gamma_{2}}, \theta \frac{\vartheta(1,2)+\nu_{7}}{\gamma_{3}} ; \Upsilon \theta\right.\right. \\
& \left.\cdot \frac{\Phi_{3}}{\gamma_{2} \gamma_{3}}\right]+N_{2}\left[\Upsilon \frac{\ln \left(\alpha_{2} S_{0}^{(2)} / K\right)+\nu_{8}}{\gamma_{5}}, \theta\right. \\
& \left.\left.\left.\cdot \frac{\vartheta(2,1)-\nu_{7}}{\gamma_{3}} ; \Upsilon \theta \frac{\Phi_{6}}{\gamma_{5} \gamma_{3}}\right]\right)\right\},
\end{aligned}
$$

where

$$
\begin{aligned}
& \Upsilon= \begin{cases}1 & \text { call option } \\
-1 & \text { put option }\end{cases} \\
& \theta= \begin{cases}1 & \text { option on maximum } \\
-1 & \text { option on minimum. }\end{cases}
\end{aligned}
$$

All the symbols and notations in Formula 1 that have not been previously defined are given in Appendix B. The same remark applies to Formulas 2 and 3.
The numerical implementation of Formula 1 is easy using the algorithm of Drezner and Wesolowsky [13], or its slightly improved version by Genz [14], to evaluate the $N_{2}[\cdot, \cdot ; \cdot]$ function with the accuracy and the efficiency required for all practical purposes. The infinite series can be truncated in a simple manner by setting a convergence threshold such that no further terms are added once the difference between two successive finite sums becomes smaller than that prespecified level. In Table 1, a few numerical results are reported. Formula 1 is applied and a comparison is made with option prices obtained in a standard Black-Scholes framework. The diffusive volatility $\sigma_{S 1}$ of asset $S^{(1)}$ is assumed to be higher than the diffusive volatility $\sigma_{S 2}$ of asset $S^{(2)}$. The intensity of the jump process $N^{(2)}$ is greater than the intensity of the jump process $N^{(1)}$. The variance of the jumps of $S^{(2)}$, that is, the parameter $\varepsilon_{2}$, is greater than the variance $\varepsilon_{1}$ of the jumps of $S^{(1)}$. Thus, the variability of $S^{(1)}$ is higher than that of $S^{(2)}$ in the continuous world, but the discontinuous variations of asset $S^{(2)}$ are more frequent and more pronounced than those of asset $S^{(1)}$. The continuous part of the correlation between $S^{(1)}$ and $S^{(2)}$, that is, the coefficient $\rho_{34}$, is negative, according to the way two-asset options are usually structured. The random factors driving the short end and the long end of the yield curve, that is, the Brownian motions $W^{(1)}$ and $W^{(2)}$, are assumed to have a positive correlation coefficient $\rho_{1.2}$. The correlation between fixed income and equity is negative, whether it is the correlation with the short end of the yield 
curve (coefficients $\rho_{1.3}$ and $\rho_{1.4}$ ) or the correlation with the long end (coefficients $\rho_{2.3}$ and $\rho_{2.4}$ ).

The values of put options on the minimum of $S^{(1)}$ and $S^{(2)}$ are computed, both at-the-money and out-of-themoney and with two different maturities. The first modeling environment considered (Model 1) is the standard BlackScholes one; that is, interest rates are constant and there are no discontinuous variations in equity asset prices. The second modeling environment considered (Model 2) is one in which the yield curve is driven by (1), but the variations of equity asset prices remain purely continuous. The other modeling environments considered (Model 3, Model 4, Model 5, and Model 6) include jumps in $S^{(1)}$ and $S^{(2)}$ according to (23) and (24), with two different levels of intensity and with or without stochastic interest rate.

For all numerical values reported in Table 1, a convergence level of $10^{-8}$ was obtained by truncating the infinite series at $n_{1}=n_{2}=n_{12}=10$, which resulted in a computational time of approximately 0.2 seconds on an ordinary personal PC. A Monte Carlo simulation algorithm was also implemented as an alternative approximate valuation method to Formula 1. It took more than two hours to achieve a $10^{-5}$ convergence level with a single value obtained by applying Formula 1. This shows how developing exact analytical formulae can result in dramatic accuracy and efficiency gains when using models implying several correlated continuous and noncontinuous sources of randomness. Monte Carlo simulation is particularly slow and inaccurate in this case, as SDEs (1), (23), and (24) need to be discretized, and the valuation algorithm has to generate a lot of interarrival times between jumps consistent with the option's specifications.

Next, the case of a spread option is handled. Lemma 5 is applied with the following inputs:

$$
\begin{aligned}
& X=\left(-\int_{0}^{T} r_{t} d t\right)+\ln \left(\frac{S_{T}^{(1)}}{S_{0}^{(1)}}\right), \\
& Y=\ln \left(\frac{S_{T}^{(1)} S_{0}^{(2)}}{S_{T}^{(2)} S_{0}^{(1)}}\right) \text { to compute } \\
& X=\left(-\int_{0}^{T} r_{t} d t\right)+\ln \left(\frac{S_{T}^{(2)}}{S_{0}^{(2)}}\right), \\
& Y=\ln \left(\frac{S_{T}^{(1)} S_{0}^{(2)}}{S_{T}^{(2)} S_{0}^{(1)}}\right) \text { to compute } \\
& X=\left(-\int_{0}^{T} r_{t} d t\right)+\ln \left(\frac{S_{T}^{(2)}}{S_{0}^{(2)}}\right), \\
& Y=\ln \left(\frac{S_{T}^{(2)} S_{0}^{(1)}}{S_{T}^{(1)} S_{0}^{(2)}}\right) \text { to compute } \\
& Y=\ln \left(\frac{S_{T}^{(2)} S_{0}^{(1)}}{S_{T}^{(1)} S_{0}^{(2)}}\right) \text { to compute } \\
& X=\left(-\int_{0}^{T} r_{t} d t\right)+\ln \left(\frac{S_{T}^{(1)}}{S_{0}^{(1)}}\right),
\end{aligned}
$$

TABLE 2: Value of a spread option ${ }^{\mathrm{a}}$.

\begin{tabular}{lcc}
\hline & $\begin{array}{c}\text { 3-month expiry } \\
\text { spread option }\end{array}$ & $\begin{array}{c}\text { 6-month expiry } \\
\text { spread option }\end{array}$ \\
\hline $\begin{array}{l}\text { Model 1: } \\
\text { Black-Scholes }\end{array}$ & 20.6206953 & 28.9473327 \\
$\begin{array}{l}\text { Model 2: low intensity } \\
\text { jumps }\end{array}$ & 21.7144031 & 30.6466976 \\
$\begin{array}{l}\text { Model 3: high } \\
\text { intensity jumps }\end{array}$ & 22.7485628 & 32.2521365 \\
\hline
\end{tabular}

$\overline{{ }^{a} \text { All values other than Black-Scholes were computed by means of Formula } 2}$ with the following inputs: $\alpha_{1}=\alpha_{2}=1 S_{0}^{(1)}=S_{0}^{(2)}=100, \sigma_{S 1}=35 \%, \sigma_{S 2}=$ $28 \%, \delta_{1}=\delta_{2}=0, \rho_{1.2}=0.3, \rho_{1.3}=-0.25, \rho_{1.4}=-0.28, \rho_{2.3}=-0.22, \rho_{2.4}=$ $-0.26, \rho_{3.4}=-0.36, \xi_{1}=\xi_{2}=0, \varepsilon_{1}=0.2$, and $\varepsilon_{2}=0.3$.

The "low intensity jumps" setting is defined by taking $\lambda_{1}^{\prime}=0.2, \lambda_{2}^{\prime}=0.3$, and $\lambda_{12}^{\prime}=0$.

The "high intensity jumps" setting is defined by taking $\lambda_{1}^{\prime}=0.4, \lambda_{2}^{\prime}=0.6$, and $\lambda_{12}^{\prime}=0$.

Deriving the required covariances (given in Appendix B), the following formula can then be obtained.

Formula 2. Let $V\left(\alpha_{1}, \alpha_{2} ; S_{0}^{(1)}, S_{0}^{(2)} ; T\right)$ denote the no-arbitrage value at time $t_{0}=0$ of a zero-strike spread option on two weighted assets $\alpha_{1} S^{(1)}$ and $\alpha_{2} S^{(2)}$ with expiry $T$. Within the modeling framework defined in Section 2, we have

$$
\begin{aligned}
& V\left(\alpha_{1}, \alpha_{2} ; S_{0}^{(1)}, S_{0}^{(2)} ; T\right)=\sum_{n_{1}=0}^{\infty} \sum_{n_{2}=0}^{\infty} \sum_{n_{12}=0}^{\infty} e^{-\lambda_{1}^{\prime} T-\lambda_{2}^{\prime} T-\lambda_{12}^{\prime} T} \\
& \cdot \frac{\left(\lambda_{1}^{\prime} T\right)^{n_{1}}\left(\lambda_{2}^{\prime} T\right)^{n_{2}}\left(\lambda_{12}^{\prime} T\right)^{n_{12}}}{n_{1} ! n_{2} ! n_{12} !}\left\{\alpha _ { 1 } S _ { 0 } ^ { ( 1 ) } \operatorname { e x p } \left(\nu_{1}\right.\right. \\
& \left.+\frac{\gamma_{1}^{2}}{2}\right) N\left[\frac{\vartheta(1,2)+\nu_{3}+\Phi_{2}}{\gamma_{3}}\right]-\alpha_{2} S_{0}^{(2)} \\
& \cdot \exp \left(\nu_{4}+\frac{\gamma_{4}^{2}}{2}\right) N\left[\frac{\vartheta(1,2)+\nu_{3}+\Phi_{7}}{\gamma_{3}}\right]+\alpha_{2} S_{0}^{(2)} \\
& \cdot \exp \left(\nu_{4}+\frac{\gamma_{4}^{2}}{2}\right) N\left[\frac{\vartheta(2,1)-\nu_{3}+\Phi_{5}}{\gamma_{3}}\right]-\alpha_{1} S_{0}^{(1)} \\
& \left.\cdot \exp \left(\nu_{1}+\frac{\gamma_{1}^{2}}{2}\right) N\left[\frac{\vartheta(2,1)-\nu_{3}+\Phi_{8}}{\gamma_{3}}\right]\right\} .
\end{aligned}
$$

The numerical implementation of Formula 2 is even easier than that of Formula 1, as only univariate standard normal cumulative distribution functions are involved instead of bivariate ones. A few numerical results are reported in Table 2. There is no model specification with a stochastic interest rate as the spread option is not a function of interest rate.

Finally, the product option is dealt with. Lemma 4 is applied to compute (21) while Lemma 5 is applied to compute 
(20) with $X=\left(-\int_{0}^{T} r_{t} d t\right)+\ln \left(S_{T}^{(1)} S_{T}^{(2)} / S_{0}^{(1)} S_{0}^{(2)}\right)$ and $Y=$ $\ln \left(S_{T}^{(1)} S_{T}^{(2)} / S_{0}^{(1)} S_{0}^{(2)}\right)$. Deriving the required covariances (given in Appendix B), the following formula can then be obtained.

Formula 3. Let $V\left(S_{0}^{(1)}, S_{0}^{(2)} ; K ; T\right)$ denote the no-arbitrage value at time $t_{0}=0$ of a product option on two assets $S^{(1)}$ and $S^{(2)}$ with strike price $K$ and expiry $T$. Within the modeling framework defined in Section 2, we have

$$
\begin{aligned}
& V\left(S_{0}^{(1)}, S_{0}^{(2)} ; K ; T\right)=\sum_{n_{1}=0}^{\infty} \sum_{n_{2}=0}^{\infty} \sum_{n_{12}=0}^{\infty} e^{-\lambda_{1}^{\prime} T-\lambda_{2}^{\prime} T-\lambda_{12}^{\prime} T} \\
& \cdot \frac{\left(\lambda_{1}^{\prime} T\right)^{n_{1}}\left(\lambda_{2}^{\prime} T\right)^{n_{2}}\left(\lambda_{12}^{\prime} T\right)^{n_{12}}}{n_{1} ! n_{2} ! n_{12} !}\left\{\Upsilon S_{0}^{(1)} S_{0}^{(2)}\right. \\
& \cdot \exp \left(v_{9}+\frac{\gamma_{6}^{2}}{2}\right) N\left[\Upsilon \frac{\ln \left(S_{0}^{(1)} S_{0}^{(2)} / K\right)+\nu_{10}+\Phi_{9}}{\gamma_{7}}\right] \\
& \left.-\Upsilon K B(0, T) N\left[\Upsilon \frac{\ln \left(S_{0}^{(1)} S_{0}^{(2)} / K\right)+\nu_{11}}{\gamma_{7}}\right]\right\} \\
& \Upsilon= \begin{cases}1 & \text { call option } \\
-1 & \text { put option. }\end{cases}
\end{aligned}
$$

\section{Conclusion}

In this article, three of the most widely traded two-asset options are analytically valued in a modeling framework allowing discontinuous variations in the equity prices, stochastic two-factor evolution of the yield curve, and crosscorrelation between all the random factors. The method used can be applied just as easily to other payoffs involving two assets, as long as they are not path-dependent (e.g., barrier or lookback option) or of American type (i.e., the option can only be exercised at expiry). For instance, one could easily handle an option based on a weighted average of the returns of two stocks at expiry; such a payoff belongs to a category known as "basket options." The same method can also be applied to options written on more than two assets, by extending Lemma 3 to a vector space of dimension greater than four. One important point should be noticed, though, when extending the method to more assets: some options, such as the product option, will still admit valuation formulae in terms of linear combinations of univariate or bivariate cumulative distribution functions, whose numerical evaluation is easy, but others, such as the option on the maximum or on the minimum, will admit valuation formulae in terms of multivariate normal cumulative distribution functions of increasing dimension, which rapidly become uneasy to evaluate with accuracy and efficiency. Also, the number of summation operators will increase, thus slowing down computational time. Hence, the extension to options written on a higher number of assets is feasible but more or less advisable, depending on the computed payoff.

\section{Appendix}

\section{A. Proofs of Lemmas}

Proof of Lemma 1.

$$
\begin{aligned}
E & {\left[\widetilde{S}_{T} \mid \mathscr{F}_{t}\right] } \\
& =E\left[\exp \left(-\int_{0}^{t} r_{s} d s\right) S_{t} \exp \left(-\int_{t}^{T}\left(r_{s}-\mu_{s}\right) d s\right)\right. \\
& \left.-\frac{\sigma^{2}}{2}(T-t)+\sigma\left(W_{T}-W_{t}\right) \prod_{i=N_{t}+1}^{N_{T}}\left(1+U_{i}\right) \mid \mathscr{F}_{t}\right] \\
& =\widetilde{S}_{t} E\left[\exp \left(-\int_{t}^{T}\left(r_{s}-\mu_{s}\right) d s\right)-\frac{\sigma^{2}}{2}(T-t)\right. \\
& \left.+\sigma\left(W_{T}-W_{t}\right) \prod_{i=1}^{N_{T}-N_{t}}\left(1+U_{N_{t}+i}\right)\right]=\widetilde{S}_{t} \\
& \cdot \exp \left(-\int_{t}^{T}\left(r_{s}-\mu_{s}\right) d s\right) E\left[\prod_{i=N_{t}+1}^{N_{T}}\left(1+U_{i}\right)\right]=\widetilde{S}_{t} \\
& \cdot \exp \left(-\int_{t}^{T}\left(r_{s}-\mu_{s}\right) d s+\lambda(T-t) E\left[U_{i}\right]\right)=\widetilde{S}_{t} \\
& \cdot \exp \left(-\int_{t}^{T}\left(r_{s}-\mu_{s}\right) d s+\lambda(T-t) \kappa\right) .
\end{aligned}
$$

Proof of Lemma 2. Since the processes $\left\{Y_{s}^{(1)}, s \geq 0\right\}$ and $\left\{Y_{s}^{(2)}, s \geq 0\right\}$ are square integrable and predictable with respect to $\mathscr{F}_{s}$, the integrals $\int_{0}^{t} Y_{s}^{(1)} d W_{s}^{(1)}$ and $\int_{0}^{t} Y_{s}^{(2)} d W_{s}^{(2)}$ are Ito integrals, and they are martingales with respect to $\mathscr{F}_{s}$. Therefore, we have

$$
\begin{aligned}
\operatorname{cov} & {\left[\int_{0}^{t} Y_{s}^{(1)} d W_{s}^{(1)}, \int_{0}^{T} Y_{s}^{(2)} d W_{s}^{(2)}\right] } \\
= & E\left[\int_{0}^{t} Y_{s}^{(1)} d W_{s}^{(1)}\right. \\
& \left.\cdot\left(\int_{0}^{t} Y_{s}^{(2)} d W_{s}^{(2)}+\int_{t}^{T} Y_{s}^{(2)} d W_{s}^{(2)}\right)\right] .
\end{aligned}
$$

By conditioning with respect to $\mathscr{F}_{t}$ and using elementary properties of the Ito integral, we obtain

$$
\begin{aligned}
E & {\left[\int_{0}^{t} Y_{s}^{(1)} d W_{s}^{(1)} \int_{t}^{T} Y_{s}^{(2)} d W_{s}^{(2)}\right] } \\
& =E\left[E\left[\int_{0}^{t} Y_{s}^{(1)} d W_{s}^{(1)} \int_{t}^{T} Y_{s}^{(2)} d W_{s}^{(2)} \mid \mathscr{F}_{t}\right]\right] \\
& =E\left[\int_{0}^{t} Y_{s}^{(1)} d W_{s}^{(1)} E\left[\int_{t}^{T} Y_{s}^{(2)} d W_{s}^{(2)} \mid \mathscr{F}_{t}\right]\right] \\
& =E\left[\int_{0}^{t} Y_{s}^{(1)} d W_{s}^{(1)} E\left[\int_{t}^{T} Y_{s}^{(2)} d W_{s}^{(2)}\right]\right]=0 .
\end{aligned}
$$


Since $\left\{Y_{s}^{(1)}, s \geq 0\right\}$ and $\left\{Y_{s}^{(2)}, s \geq 0\right\}$ are right-continuous, square integrable processes, they can be approximated, respectively, by the following simple processes:

$$
\begin{aligned}
& \widetilde{Y}_{s}^{(1)}=\sum_{i=0}^{n-1} \phi_{i}^{(1)} \rrbracket_{\left(t_{i}, t_{i+1}\right]}(s) \\
& \widetilde{Y}_{s}^{(2)}=\sum_{i=1}^{n-1} \phi_{i}^{(2)} \rrbracket_{\left(t_{i}, t_{i+1}\right]}(s),
\end{aligned}
$$

where $n$ is the number of points in a sequence of partitions of $\left[0=t_{0}, t=t_{n}\right] ; \phi_{i}^{(1)}$ and $\phi_{i}^{(2)}$ are constants if $\left(Y^{(1)}, Y^{(2)}\right)$ is a pair of deterministic processes; $\phi_{i}^{(1)}$ and $\phi_{i}^{(2)}$ are square integrable, $\mathscr{F}_{t_{i}}$-adapted, random variables if $\left(Y^{(1)}, Y^{(2)}\right)$ is a pair of random processes; $\phi_{t_{0}}^{(1)}=Y_{t_{0}}^{(1)}$ and $\phi_{t_{0}}^{(2)}=Y_{t_{0}}^{(2)}$.

It is a classical result from the theory of continuoustime processes that $\int_{0}^{t} Y_{s}^{(1)} d W_{s}^{(1)}$ and $\int_{0}^{t} Y_{s}^{(2)} d W_{s}^{(2)}$ can be approximated by

$$
\begin{gathered}
\lim _{n \rightarrow \infty} \sum_{i=0}^{n-1} \phi_{i}^{(1)}\left(W_{t_{i+1}}^{(1)}-W_{t_{i}}^{(1)}\right) \\
\lim _{n \rightarrow \infty} \sum_{i=0}^{n-1} \phi_{i}^{(2)}\left(W_{t_{i+1}}^{(2)}-W_{t_{i}}^{(2)}\right),
\end{gathered}
$$

By conditioning on $\mathscr{F}_{t_{i} \wedge t_{j}}$, the sum $S_{2}$ can be rewritten as follows:

respectively, where the approximating sums in (A.5) converge in the mean square sense [15]. Hence, as $n \rightarrow \infty$,

$$
\begin{aligned}
E & {\left[\int_{0}^{t} Y_{s}^{(1)} d W_{s}^{(1)} \int_{0}^{t} Y_{s}^{(2)} d W_{s}^{(2)}\right]=E\left[\sum _ { i = 0 } ^ { n - 1 } \phi _ { i } ^ { ( 1 ) } \left(W_{t_{i+1}}^{(1)}\right.\right.} \\
& \left.\left.-W_{t_{i}}^{(1)}\right) \sum_{j=0}^{n-1} \phi_{j}^{(2)}\left(W_{t_{j+1}}^{(2)}-W_{t_{j}}^{(2)}\right)\right] \\
& =\sum_{i=0}^{n-1} E\left[\phi_{i}^{(1)} \phi_{i}^{(2)}\left(W_{t_{i+1}}^{(1)}-W_{t_{i}}^{(1)}\right)\left(W_{t_{i+1}}^{(2)}-W_{t_{i}}^{(2)}\right)\right] \\
& +\sum_{i=0}^{n-1} \sum_{\substack{j=0 \\
j \neq i}}^{n-1} E\left[\phi_{i}^{(1)} \phi_{j}^{(2)}\left(W_{t_{i+1}}^{(1)}-W_{t_{i}}^{(1)}\right)\right. \\
& \left.\cdot\left(W_{t_{j+1}}^{(2)}-W_{t_{j}}^{(2)}\right)\right] \triangleq S_{1}+S_{2} .
\end{aligned}
$$

$$
\begin{aligned}
S_{2}= & \sum_{i=0}^{n-1} \sum_{\substack{j=0 \\
j \neq i}}^{n-1} E\left[E\left[\phi_{i}^{(1)} \phi_{j}^{(2)}\left(W_{t_{i+1}}^{(1)}-W_{t_{i}}^{(1)}\right)\left(W_{t_{j+1}}^{(2)}-W_{t_{j}}^{(2)}\right) \mid \mathscr{F}_{t_{i} \wedge t_{j}}\right]\right] \\
= & \sum_{i=0}^{n-1} \sum_{\substack{j=0 \\
j \neq i}}^{n-1} E\left[\phi_{i}^{(1)} E\left[\phi_{j}^{(2)} \mid \mathscr{F}_{t_{i}}\right] E\left[\left(W_{t_{i+1}}^{(1)}-W_{t_{i}}^{(1)}\right)\left(W_{t_{j+1}}^{(2)}-W_{t_{j}}^{(2)}\right)\right]\right] \mathbb{q}_{\left\{t_{i}<t_{j}\right\}} \\
& +\sum_{i=0}^{n-1} \sum_{\substack{j=0 \\
j \neq i}}^{n-1} E\left[\phi_{j}^{(2)} E\left[\phi_{i}^{(1)} \mid \mathscr{F}_{t_{j}}\right] E\left[\left(W_{t_{i+1}}^{(1)}-W_{t_{i}}^{(1)}\right)\left(W_{t_{j+1}}^{(2)}-W_{t_{j}}^{(2)}\right)\right]\right] \mathbb{\{}_{\left\{t_{j}<t_{i}\right\}} .
\end{aligned}
$$

Denoting by $\left\{\bar{W}_{t}^{(2)}, t \geq 0\right\}$ a standard Brownian motion independent of $\left\{W_{t}^{(1)}, t \geq 0\right\}$ defined on the same filtered probability space as $\left\{W_{t}^{(2)}, t \geq 0\right\}$, one can apply the wellknown orthogonal decomposition of $W^{(2)}$ with respect to $W^{(1)}$ in the following manner:

$$
\begin{gathered}
E\left[\left(W_{t_{i+1}}^{(1)}-W_{t_{i}}^{(1)}\right)\left(W_{t_{j+1}}^{(2)}-W_{t_{j}}^{(2)}\right)\right]=E\left[\left(W_{t_{i+1}}^{(1)}\right.\right. \\
\left.-W_{t_{i}}^{(1)}\right)\left(\rho W_{t_{j+1}}^{(1)}+\sqrt{1-\rho^{2}} \bar{W}_{t_{j+1}}^{(2)}-\rho W_{t_{j}}^{(1)}\right. \\
\left.\left.-\sqrt{1-\rho^{2}} \bar{W}_{t_{j}}^{(2)}\right)\right]=\rho \operatorname{cov}\left[W_{t_{i+1}}^{(1)}, W_{t_{j+1}}^{(1)}\right]-\rho \\
\cdot \operatorname{cov}\left[W_{t_{i+1}}^{(1)}, W_{t_{j}}^{(1)}\right]-\rho \operatorname{cov}\left[W_{t_{i}}^{(1)}, W_{t_{j+1}}^{(1)}\right]+\rho \\
\cdot \operatorname{cov}\left[W_{t_{i}}^{(1)}, W_{t_{j}}^{(1)}\right] .
\end{gathered}
$$

If $i$ and $j$ are two natural integers such that $i<j$, then $\sup _{i}((i+$ $1)-j)=0$; similarly, if $j<i$, then $\sup _{j}((j+1)-i)=0$. Thus,

$$
\begin{aligned}
E[ & \left.\left(W_{t_{i+1}}^{(1)}-W_{t_{i}}^{(1)}\right)\left(W_{t_{j+1}}^{(2)}-W_{t_{j}}^{(2)}\right)\right] \\
= & \rho\left(t_{i+1}-t_{i+1}-t_{i}+t_{i}\right) \mathbb{a}_{\{i<j\}} \\
& +\rho\left(t_{j+1}-t_{j}-t_{j+1}+t_{j}\right) \mathbb{q}_{\{i>j\}}=0 .
\end{aligned}
$$

Hence, $S_{2}=0$. Turning to the sum $S_{1}$, we obtain

$$
\begin{aligned}
& S_{1}=\sum_{i=0}^{n-1} E\left[E \left[\phi_{i}^{(1)} \phi_{i}^{(2)}\left(W_{t_{i+1}}^{(1)}-W_{t_{i}}^{(1)}\right)\left(W_{t_{i+1}}^{(2)}-W_{t_{i}}^{(2)}\right) \mid\right.\right. \\
&\left.\left.\mathscr{F}_{t_{i}}\right]\right]=\sum_{i=0}^{n-1} E\left[\phi _ { i } ^ { ( 1 ) } \phi _ { i } ^ { ( 2 ) } E \left[\left(W_{t_{i+1}}^{(1)}-W_{t_{i}}^{(1)}\right)\right.\right.
\end{aligned}
$$




$$
\begin{aligned}
& \left.\left.\cdot\left(\rho W_{t_{i+1}}^{(1)}+\sqrt{1-\rho^{2}} \bar{W}_{t_{i+1}}^{(2)}-\rho W_{t_{i}}^{(1)}-\sqrt{1-\rho^{2}} \bar{W}_{t_{i}}^{(2)}\right)\right]\right] \\
& =\sum_{i=0}^{n-1} E\left[\phi_{i}^{(1)} \phi_{i}^{(2)} \rho\left(t_{i+1}-t_{i}\right)\right]=\rho \sum_{i=0}^{n-1} E\left[\phi_{i}^{(1)} \phi_{i}^{(2)}\right]\left(t_{i+1}\right. \\
& \left.-t_{i}\right),
\end{aligned}
$$

where the last sum converges in mean square to $\rho \int_{0}^{t} E\left[Y_{s}^{(1)} Y_{s}^{(2)}\right] d s$.

Proof of Lemma 3. Equation (32) is a well-known result, used already in the proof of Lemma 2. Since any finite-dimensional Gaussian space is a vector space with respect to a basis of pairwise independent standard normal Gaussian random variables, the random variable $W_{t}^{(3)}$ admits the following orthogonal decomposition:

$$
W_{t}^{(3)}=\rho_{1.3} W_{t}^{(1)}+a_{2} \bar{W}_{t}^{(2)}+a_{3} \bar{W}_{t}^{(3)}(t),
$$

where $a_{2}$ and $a_{3}$ are real-valued scalars; $a_{3}$ must be positive since, by definition of the multivariate normal random vector $\left[W_{t}^{(1)}, W_{t}^{(2)}, W_{t}^{(3)}\right], a_{3} \sqrt{t}$ has to be the standard deviation of $W_{t}^{(3)}$ conditional on $W_{t}^{(1)}$ and $W_{t}^{(2)}$. From the definition of linear correlation and the bilinearity of covariance, we obtain

$$
\begin{aligned}
\rho_{2.3} & =\frac{\operatorname{cov}\left[W_{t}^{(2)}, W_{t}^{(3)}\right]}{t} \\
& =\frac{1}{t}\left(\operatorname{cov}\left[\rho_{1.2} W_{t}^{(1)}, \rho_{1.3} W_{t}^{(1)}\right]\right. \\
& \left.+\operatorname{cov}\left[\sigma_{2 \mid 1} \bar{W}_{t}^{(2)}, a_{2} \bar{W}_{t}^{(2)}\right]\right) \longleftrightarrow a_{2}=\rho_{2.3 \mid 1} .
\end{aligned}
$$

From the standard deviation of $W_{t}^{(3)}$ and from the stability under addition of the normal distribution, we get

$$
\sqrt{t\left(\rho_{1.3}^{2}+\rho_{2.3 \mid 1}^{2}+a_{3}^{2}\right)}=\sqrt{t} \longleftrightarrow a_{3}=\sigma_{3 \mid 1.2} .
$$

The orthogonal decomposition of $W_{t}^{(4)}$ in (34) is derived in a similar manner.

Proof of Lemma 4. From (30) and from the formula for the moment generating function of a normal random variable, we get $B(t, T)$ as given by Lemma $4 ; B(t, T)$ is the price, at time $t$, of a zero-coupon bond maturing at $T$. Applying Ito's lemma to $\ln (B(t, T))$ under $Q$ and then integrating on $[0, t]$, one can obtain

$$
B(t, T)=B(0, T) \beta_{t} L(t, T)
$$

with

$$
\begin{aligned}
& L(t, T)=\exp \left(-\frac{1}{2}\right. \\
& \cdot \int_{0}^{t}\left(A^{2}(s, T)\left(\sigma_{R 1}^{2}+\sigma_{R 2}^{2}+2 \sigma_{R 1} \sigma_{R 2} \rho_{1.2}\right)\right) d s \\
& -\left(\sigma_{R 1}+\sigma_{R 2} \rho_{1.2}\right) \\
& \left.\cdot \int_{0}^{t} A(s, T) d W_{s}^{(1)}-\sigma_{R 2} \sigma_{2 \mid 1} \int_{0}^{t} A(s, T) d \bar{W}_{s}^{(2)}\right) .
\end{aligned}
$$

Hence, we have

$$
\begin{aligned}
E_{\mathrm{Q}} & {\left[\exp \left(-\int_{0}^{T} r_{t} d t\right) \square\{\mathscr{Z}\} \mid \mathscr{F}_{0}\right] } \\
= & E_{\mathrm{Q}}\left[\frac{B(t, T)}{\beta_{t}} \square\{\mathscr{E}\} \mid \mathscr{F}_{0}\right] \\
= & B(0, T) E_{P_{B_{T}}}\left[\square\{\mathscr{E}\} \mid \mathscr{F}_{0}\right],
\end{aligned}
$$

where $P_{B_{T}}$ is the equivalent martingale measure under which the numeraire is the zero-coupon bond, whose RadonNikodym derivative is given by

$$
\frac{d P_{B_{T}}}{d Q} \mid \mathscr{F}_{t}=L(t, T) .
$$

Proof of Lemma 5. Let $f_{X, Y, Z}$ denote the joint density of $X, Y$, and $Z$. Then,

$$
\begin{aligned}
E & {[\exp (X) \square\{Y \leq a, Z \leq b\}]=\int_{x=-\infty}^{\infty} \int_{y=-\infty}^{a} \int_{z=-\infty}^{b} e^{x} f_{X, Y, Z}(x, y, z) d z d y d x } \\
& =\int_{x=-\infty}^{\infty} \int_{y=-\infty}^{a} \int_{z=-\infty}^{b}\left\{\frac{\exp \left(x-(1 / 2)\left(\left(x-\mu_{X}\right) / \sigma_{X}\right)^{2}-\left(1 / 2 \sigma_{Y \mid X}^{2}\right)\left(\left(y-\mu_{Y}\right) / \sigma_{Y}-\rho_{X . Y}\left(\left(x-\mu_{X}\right) / \sigma_{X}\right)\right)^{2}\right)}{2 \pi \sigma_{X} \sigma_{Y} \sigma_{Y \mid X}}\right. \\
& \left.\cdot \frac{\exp \left(-\left(1 / 2 \sigma_{Z \mid X . Y}^{2}\right)\left(\left(z-\mu_{Z}\right) / \sigma_{Z}-\rho_{X . Z}\left(\left(x-\mu_{X}\right) / \sigma_{X}\right)-\rho_{Y . Z \mid X}\left(\left(1 / \sigma_{Y \mid X}\right)\left(\left(y-\mu_{Y}\right) / \sigma_{Y}-\rho_{X . Y}\left(\left(x-\mu_{X}\right) / \sigma_{X}\right)\right)\right)\right)^{2}\right)}{\sigma_{Z} \sigma_{Z \mid X . Y} \sqrt{2 \pi}}\right\} d z d y d x .
\end{aligned}
$$

Apply the following chain of changes of variables:

$$
\begin{aligned}
& \tilde{x}=\frac{x-\mu_{X}}{\sigma_{X}}, \\
& \tilde{y}=\frac{y-\mu_{Y}}{\sigma_{Y}},
\end{aligned}
$$

$$
\begin{aligned}
& \hat{x}=\tilde{x}-\sigma_{X}, \\
& \hat{y}=\tilde{y}-\rho_{X . Y} \sigma_{X}, \\
& \tilde{z}=\frac{z-\mu_{Z}}{\sigma_{Z}}-\rho_{X . Z} \sigma_{X},
\end{aligned}
$$


12

Journal of Applied Mathematics

and then use the identity

$$
\int_{-\infty}^{\infty} f_{X, Y, Z}(x, y, z) d x=f_{Y, Z}(y, z)
$$

to obtain Lemma 5.

$$
\begin{aligned}
& \nu_{1}=E_{\mathrm{Q}}\left[\left(-\int_{0}^{T} r_{t} d t\right)+\ln \left(\frac{S_{T}^{(1)}}{S_{0}^{(1)}}\right)\right]=\left(n_{1}+n_{12}\right) \xi_{1} \\
& -\left(\lambda_{1} \kappa_{1}+\delta_{1}+\frac{\sigma_{S 1}^{2}}{2}\right) T \\
& v_{2}=E_{\mathrm{Q}}\left[\ln \left(\frac{S_{T}^{(1)}}{S_{0}^{(1)}}\right)\right]=\bar{\mu}_{r}+v_{1} \\
& \nu_{3}=E_{\mathrm{Q}}\left[\ln \left(\frac{S_{T}^{(1)} S_{0}^{(2)}}{S_{T}^{(2)} S_{0}^{(1)}}\right)\right]=-E_{\mathrm{Q}}\left[\ln \left(\frac{S_{T}^{(2)} S_{0}^{(1)}}{S_{T}^{(1)} S_{0}^{(2)}}\right)\right] \\
& =\left(\delta_{2}-\delta_{1}+\lambda_{2} \kappa_{2}-\lambda_{1} \kappa_{1}+\frac{\sigma_{S 2}^{2}-\sigma_{S 1}^{2}}{2}\right) T+\left(n_{1}\right. \\
& \left.+n_{12}\right) \xi_{1}-\left(n_{2}+n_{12}\right) \xi_{2} \\
& v_{4}=E_{\mathrm{Q}}\left[\left(-\int_{0}^{T} r_{t} d t\right)+\ln \left(\frac{S_{T}^{(2)}}{S_{0}^{(2)}}\right)\right]=\left(n_{2}+n_{12}\right) \xi_{2} \\
& -\left(\lambda_{2} \kappa_{2}+\delta_{2}+\frac{\sigma_{S 2}^{2}}{2}\right) T \\
& v_{5}=E_{Q}\left[\ln \left(\frac{S_{T}^{(2)}}{S_{0}^{(2)}}\right)\right]=\bar{\mu}_{r}+v_{4} \\
& \nu_{8}=E_{P_{B_{T}}}\left[\ln \left(\frac{S_{T}^{(2)}}{S_{0}^{(2)}}\right)\right]=\bar{\mu}_{r}-\left(\delta_{2}+\lambda_{2} \kappa_{2}+\frac{\sigma_{S 2}^{2}}{2}\right) T \\
& +\left(n_{2}+n_{12}\right) \xi_{2}+\frac{1-e^{-a T}-T}{a^{2}}\left(\left(\sigma_{R 1}+\sigma_{R 2} \rho_{1.2}\right)\right. \\
& \cdot\left(\bar{\sigma}_{r}\left(\sigma_{R 1}+\sigma_{R 2} \rho_{1.2}\right)+\sigma_{S 2} \rho_{1.4}\right) \\
& \left.+\sigma_{R 2} \sigma_{2 \mid 1}\left(\bar{\sigma}_{r} \sigma_{R 2} \sigma_{2 \mid 1}+\sigma_{S 2} \rho_{2.4 \mid 1}\right)\right) \\
& \nu_{9}=E_{\mathrm{Q}}\left[\left(-\int_{0}^{T} r_{t} d t\right)+\ln \left(\frac{S_{T}^{(1)} S_{T}^{(2)}}{S_{0}^{(1)} S_{0}^{(2)}}\right)\right]=\bar{\mu}_{r}-\left(\delta_{1}\right. \\
& \left.+\delta_{2}+\lambda_{1} \kappa_{1}+\lambda_{2} \kappa_{2}+\frac{\sigma_{S 1}^{2}+\sigma_{S 2}^{2}}{2}\right) T+\left(n_{1}+n_{12}\right) \xi_{1} \\
& +\left(n_{2}+n_{12}\right) \xi_{2} \\
& \nu_{10}=E_{\mathrm{Q}}\left[\ln \left(\frac{S_{T}^{(1)} S_{T}^{(2)}}{S_{0}^{(1)} S_{0}^{(2)}}\right)\right]=\nu_{9}+\bar{\mu}_{r} \\
& v_{11}=E_{P_{B_{T}}}\left[\ln \left(\frac{S_{T}^{(1)} S_{T}^{(2)}}{S_{0}^{(1)} S_{0}^{(2)}}\right)\right]=2 \bar{\mu}_{r}-\left(\delta_{1}+\delta_{2}\right. \\
& \left.+\lambda_{1} \kappa_{1}+\lambda_{2} \kappa_{2}+\frac{\sigma_{S 1}^{2}+\sigma_{S 2}^{2}}{2}\right) T \\
& +\frac{\left(\sigma_{R 1}+\sigma_{R 2} \rho_{1.2}\right)\left(1-e^{-a T}-T\right)}{a^{2}}\left(2 \overline { \sigma } _ { r } \left(\sigma_{R 1}\right.\right. \\
& \left.\left.+\sigma_{R 2} \rho_{1.2}\right)+\sigma_{S 1} \rho_{1.3}+\sigma_{S 2} \rho_{1.4}\right) \\
& +\frac{\sigma_{R 2} \sigma_{2 \mid 1}\left(1-e^{-a T}-T\right)}{a^{2}}\left(2 \bar{\sigma}_{r} \sigma_{R 2} \sigma_{2 \mid 1}+\sigma_{S 1} \rho_{2.3 \mid 1}\right. \\
& \left.+\sigma_{S 2} \rho_{2.4 \mid 1}\right)+\left(n_{1}+n_{12}\right) \xi_{1}+\left(n_{2}+n_{12}\right) \xi_{2} .
\end{aligned}
$$$$
v_{6}=E_{P_{B_{T}}}\left[\ln \left(\frac{S_{T}^{(1)}}{S_{0}^{(1)}}\right)\right]=\bar{\mu}_{r}-\left(\delta_{1}+\lambda_{1} \kappa_{1}+\frac{\sigma_{S 1}^{2}}{2}\right) T
$$$$
+\left(n_{1}+n_{12}\right) \xi_{1}+\frac{1-e^{-a T}-T}{a^{2}}\left(\left(\sigma_{R 1}+\sigma_{R 2} \rho_{1.2}\right)\right.
$$$$
\cdot\left(\bar{\sigma}_{r}\left(\sigma_{R 1}+\sigma_{R 2} \rho_{1.2}\right)+\sigma_{S 1} \rho_{1.3}\right)
$$$$
\left.+\sigma_{R 2} \sigma_{2 \mid 1}\left(\bar{\sigma}_{r} \sigma_{R 2} \sigma_{2 \mid 1}+\sigma_{S 1} \rho_{2.3 \mid 1}\right)\right)
$$$$
v_{7}=E_{P_{B_{T}}}\left[\ln \left(\frac{S_{T}^{(1)} S_{0}^{(2)}}{S_{T}^{(2)} S_{0}^{(1)}}\right)\right]=-E_{P_{B_{T}}}\left[\ln \left(\frac{S_{T}^{(2)} S_{0}^{(1)}}{S_{T}^{(1)} S_{0}^{(2)}}\right)\right]
$$$$
=\frac{1-e^{-a T}-T}{a^{2}}\left(\left(\sigma_{R 1}+\sigma_{R 2} \rho_{1.2}\right)\left(\sigma_{S 1} \rho_{1.3}-\sigma_{S 2} \rho_{1.4}\right)\right.
$$

$$
\begin{aligned}
& \left.+\sigma_{R 2} \sigma_{2 \mid 1}\left(\sigma_{S 1} \rho_{2.3 \mid 1}-\sigma_{S 2} \rho_{2.4 \mid 1}\right)\right)+\left(\delta_{2}-\delta_{1}\right. \\
& \left.+\lambda_{2} \kappa_{2}-\lambda_{1} \kappa_{1}+\frac{\left(\sigma_{S 2}^{2}-\sigma_{S 1}^{2}\right)}{2}\right) T+\left(n_{1}+n_{12}\right) \xi_{1} \\
& -\left(n_{2}+n_{12}\right) \xi_{2}
\end{aligned}
$$

(2) The $\gamma$-Terms. This collection of terms refers to standard deviations involved in Formulas 1, 2, and 3:

$$
\begin{aligned}
\gamma_{1} & =\left(\operatorname{var}\left[\left(-\int_{0}^{T} r_{t} d t\right)+\ln \left(\frac{S_{T}^{(1)}}{S_{0}^{(1)}}\right)\right]\right)^{1 / 2}=\left(\sigma_{S 1}^{2} T\right. \\
& \left.+\left(n_{1}+n_{12}\right) \varepsilon_{1}^{2}\right)^{1 / 2} \\
\gamma_{2} & =\left(\operatorname{var}\left[\ln \left(\frac{S_{T}^{(1)}}{S_{0}^{(1)}}\right)\right]\right)^{1 / 2} \\
& =\left(\left(\left(\bar{\sigma}_{r}\left(\sigma_{R 1}+\sigma_{R 2} \rho_{1.2}\right)+\sigma_{S 1} \rho_{1.3}\right)^{2}\right.\right.
\end{aligned}
$$


Journal of Applied Mathematics

13

$$
\begin{aligned}
& \left.+\left(\bar{\sigma}_{r} \sigma_{R 2} \sigma_{2 \mid 1}+\sigma_{S 1} \rho_{2.3 \mid 1}\right)^{2}+\left(\sigma_{S 1} \sigma_{3 \mid 1.2}\right)^{2}\right) T+\left(n_{1}\right. \\
& \left.\left.+n_{12}\right) \varepsilon_{1}^{2}\right)^{1 / 2} \\
& \gamma_{3}=\left(\operatorname{var}\left[\ln \left(\frac{S_{T}^{(1)} S_{0}^{(2)}}{S_{T}^{(2)} S_{0}^{(1)}}\right)\right]\right)^{1 / 2} \\
& =\left(\operatorname{var}\left[\ln \left(\frac{S_{T}^{(2)} S_{0}^{(1)}}{S_{T}^{(1)} S_{0}^{(2)}}\right)\right]\right)^{1 / 2} \\
& =\left(\left(\left(\sigma_{S 1} \rho_{1.3}-\sigma_{S 2} \rho_{1.4}\right)^{2}+\left(\sigma_{S 1} \rho_{2.3 \mid 1}-\sigma_{S 2} \rho_{2.4 \mid 1}\right)^{2}\right.\right. \\
& \left.+\left(\sigma_{S 1} \sigma_{3 \mid 1.2}-\sigma_{S 2} \rho_{3.4 \mid 1.2}\right)^{2}+\left(\sigma_{S 2} \sigma_{4 \mid 1.2 .3}\right)^{2}\right) T+\left(n_{1}\right. \\
& \left.\left.+n_{12}\right) \varepsilon_{1}^{2}+\left(n_{2}+n_{12}\right) \varepsilon_{2}^{2}\right)^{1 / 2} \\
& \gamma_{4}=\left(\operatorname{var}\left[\left(-\int_{0}^{T} r_{t} d t\right)+\ln \left(\frac{S_{T}^{(2)}}{S_{0}^{(2)}}\right)\right]\right)^{1 / 2}=\left(\sigma_{S 2}^{2} T\right. \\
& \left.+\left(n_{2}+n_{12}\right) \varepsilon_{2}^{2}\right)^{1 / 2} \\
& \gamma_{5}=\left(\operatorname{var}\left[\ln \left(\frac{S_{T}^{(2)}}{S_{0}^{(2)}}\right)\right]\right)^{1 / 2} \\
& =\left(\left(\left(\bar{\sigma}_{r}\left(\sigma_{R 1}+\sigma_{R 2} \rho_{1.2}\right)+\sigma_{S 2} \rho_{1.4}\right)^{2}\right.\right. \\
& +\left(\bar{\sigma}_{r} \sigma_{R 2} \sigma_{2 \mid 1}+\sigma_{S 2} \rho_{2.4 \mid 1}\right)^{2}+\left(\sigma_{S 2} \rho_{3.4 \mid 1.2}\right)^{2} \\
& \left.\left.+\left(\sigma_{S 2} \sigma_{4 \mid 1.2 .3}\right)^{2}\right) T+\left(n_{2}+n_{12}\right) \varepsilon_{2}^{2}\right)^{1 / 2} \\
& \gamma_{6}=\left(\operatorname{var}\left[\left(-\int_{0}^{T} r_{t} d t\right)+\left[\ln \left(\frac{S_{T}^{(1)} S_{T}^{(2)}}{S_{0}^{(1)} S_{0}^{(2)}}\right)\right]\right]\right)^{1 / 2} \\
& =\left(\left(\left(\bar{\sigma}_{r}\left(\sigma_{R 1}+\sigma_{R 2} \rho_{1.2}\right)+\sigma_{S 1} \rho_{1.3}+\sigma_{S 2} \rho_{1.4}\right)^{2}\right.\right. \\
& +\left(\bar{\sigma}_{r} \sigma_{R 2} \sigma_{2 \mid 1}+\sigma_{S 1} \rho_{2.3 \mid 1}+\sigma_{S 2} \rho_{2.4 \mid 1}\right)^{2} \\
& \left.+\left(\sigma_{S 1} \sigma_{3 \mid 1.2}+\sigma_{S 2} \rho_{3.4 \mid 1.2}\right)^{2}+\left(\sigma_{S 2} \sigma_{4 \mid 1.2 .3}\right)^{2}\right) T+\left(n_{1}\right. \\
& \left.\left.+n_{12}\right) \varepsilon_{1}^{2}+\left(n_{2}+n_{12}\right) \varepsilon_{2}^{2}\right)^{1 / 2} \\
& \gamma_{7}=\left(\operatorname{var}\left[\ln \left(\frac{S_{T}^{(1)} S_{T}^{(2)}}{S_{0}^{(1)} S_{0}^{(2)}}\right)\right]\right)^{1 / 2} \\
& =\left(\left(\left(2 \bar{\sigma}_{r}\left(\sigma_{R 1}+\sigma_{R 2} \rho_{1.2}\right)+\sigma_{S 1} \rho_{1.3}+\sigma_{S 2} \rho_{1.4}\right)^{2}\right.\right. \\
& +\left(2 \bar{\sigma}_{r} \sigma_{R 2} \sigma_{2 \mid 1}+\sigma_{S 1} \rho_{2.3 \mid 1}+\sigma_{S 2} \rho_{2.4 \mid 1}\right)^{2} \\
& \left.+\left(\sigma_{S 1} \sigma_{3 \mid 1.2}+\sigma_{S 2} \rho_{3.4 \mid 1.2}\right)^{2}+\left(\sigma_{S 2} \sigma_{4 \mid 1.2 .3}\right)^{2}\right) T+\left(n_{1}\right. \\
& \left.\left.+n_{12}\right) \varepsilon_{1}^{2}+\left(n_{2}+n_{12}\right) \varepsilon_{2}^{2}\right)^{1 / 2} \text {. } \\
& \Phi_{1}=\operatorname{cov}\left[\left(-\int_{0}^{T} r_{t} d t\right)+\ln \left(\frac{S_{T}^{(1)}}{S_{0}^{(1)}}\right), \ln \left(\frac{S_{T}^{(1)}}{S_{0}^{(1)}}\right)\right] \\
& =\left(\left(\sigma_{S 1} \rho_{1.3}\right)\left(\bar{\sigma}_{r}\left(\sigma_{R 1}+\sigma_{R 2} \rho_{1.2}\right)+\sigma_{S 1} \rho_{1.3}\right)\right. \\
& \left.+\sigma_{S 1} \rho_{2.3 \mid 1}\left(\bar{\sigma}_{r} \sigma_{R 2} \sigma_{2 \mid 1}+\sigma_{S 1} \rho_{2.3 \mid 1}\right)+\left(\sigma_{S 1} \sigma_{3 \mid 1.2}\right)^{2}\right) T \\
& +\left(n_{1}+n_{12}\right) \varepsilon_{1}^{2} \\
& \Phi_{2}=\operatorname{cov}\left[\left(-\int_{0}^{T} r_{t} d t\right)\right. \\
& \left.+\ln \left(\frac{S_{T}^{(1)}}{S_{0}^{(1)}}\right), \ln \left(\frac{S_{T}^{(1)} S_{0}^{(2)}}{S_{T}^{(2)} S_{0}^{(1)}}\right)\right]=\left(\sigma _ { S 1 } \rho _ { 1 . 3 } \left(\sigma_{S 1} \rho_{1.3}\right.\right. \\
& \left.-\sigma_{S 2} \rho_{1.4}\right)+\sigma_{S 1} \rho_{2.3 \mid 1}\left(\sigma_{S 1} \rho_{2.3 \mid 1}-\sigma_{S 2} \rho_{2.4 \mid 1}\right) \\
& \left.+\sigma_{S 1} \sigma_{3 \mid 1.2}\left(\sigma_{S 1} \sigma_{3 \mid 1.2}-\sigma_{S 2} \rho_{3.4 \mid 1.2}\right)\right) T+\left(n_{1}+n_{12}\right) \\
& \cdot \varepsilon_{1}^{2} \\
& \Phi_{3}=\operatorname{cov}\left[\ln \left(\frac{S_{T}^{(1)}}{S_{0}^{(1)}}\right), \ln \left(\frac{S_{T}^{(1)} S_{0}^{(2)}}{S_{T}^{(2)} S_{0}^{(1)}}\right)\right] \\
& =\left(( \overline { \sigma } _ { r } ( \sigma _ { R 1 } + \sigma _ { R 2 } \rho _ { 1 . 2 } ) + \sigma _ { S 1 } \rho _ { 1 . 3 } ) \left(\sigma_{S 1} \rho_{1.3}\right.\right. \\
& \left.-\sigma_{S 2} \rho_{1.4}\right)+\left(\bar{\sigma}_{r} \sigma_{R 2} \sigma_{2 \mid 1}+\sigma_{S 1} \rho_{2.3 \mid 1}\right)\left(\sigma_{S 1} \rho_{2.3 \mid 1}\right. \\
& \left.\left.-\sigma_{S 2} \rho_{2.4 \mid 1}\right)+\sigma_{S 1} \sigma_{3 \mid 1.2}\left(\sigma_{S 1} \sigma_{3 \mid 1.2}-\sigma_{S 2} \rho_{3.4 \mid 1.2}\right)\right) T \\
& +\left(n_{1}+n_{12}\right) \varepsilon_{1}^{2} \\
& \Phi_{4}=\operatorname{cov}\left[\left(-\int_{0}^{T} r_{t} d t\right)+\ln \left(\frac{S_{T}^{(2)}}{S_{0}^{(2)}}\right), \ln \left(\frac{S_{T}^{(2)}}{S_{0}^{(2)}}\right)\right] \\
& =\left(\sigma_{S 2} \rho_{1.4}\left(\bar{\sigma}_{r}\left(\sigma_{R 1}+\sigma_{R 2} \rho_{1.2}\right)+\sigma_{S 2} \rho_{1.4}\right)\right. \\
& +\sigma_{S 2} \rho_{2.4 \mid 1}\left(\bar{\sigma}_{r} \sigma_{R 2} \sigma_{2 \mid 1}+\sigma_{S 2} \rho_{2.4 \mid 1}\right)+\left(\sigma_{S 2} \rho_{3.4 \mid 1.2}\right)^{2} \\
& \left.+\left(\sigma_{S 2} \sigma_{4 \mid 1.2 .3}\right)^{2}\right) T+\left(n_{2}+n_{12}\right) \varepsilon_{2}^{2} \\
& \Phi_{5}=\operatorname{cov}\left[\left(-\int_{0}^{T} r_{t} d t\right)\right. \\
& \left.+\ln \left(\frac{S_{T}^{(2)}}{S_{0}^{(2)}}\right), \ln \left(\frac{S_{T}^{(2)} S_{0}^{(1)}}{S_{T}^{(1)} S_{0}^{(2)}}\right)\right]=\left(\sigma _ { S 2 } \rho _ { 1 . 4 } \left(\sigma_{S 2} \rho_{1.4}\right.\right. \\
& \left.-\sigma_{S 1} \rho_{1.3}\right)+\sigma_{S 2} \rho_{2.4 \mid 1}\left(\sigma_{S 2} \rho_{2.4 \mid 1}-\sigma_{S 1} \rho_{2.3 \mid 1}\right) \\
& +\sigma_{S 2} \rho_{3.4 \mid 1.2}\left(\sigma_{S 2} \rho_{3.4 \mid 1.2}-\sigma_{S 1} \sigma_{3 \mid 1.2}\right) \\
& \left.+\left(\sigma_{S 2} \sigma_{4 \mid 1.2 .3}\right)^{2}\right) T+\left(n_{2}+n_{12}\right) \varepsilon_{2}^{2} \\
& \Phi_{6}=\operatorname{cov}\left[\ln \left(\frac{S_{T}^{(2)}}{S_{0}^{(2)}}\right), \ln \left(\frac{S_{T}^{(2)} S_{0}^{(1)}}{S_{T}^{(1)} S_{0}^{(2)}}\right)\right]
\end{aligned}
$$




$$
\begin{aligned}
& =\left(( \overline { \sigma } _ { r } ( \sigma _ { R 1 } + \sigma _ { R 2 } \rho _ { 1 . 2 } ) + \sigma _ { S 2 } \rho _ { 1 . 4 } ) \left(\sigma_{S 2} \rho_{1.4}\right.\right. \\
& \left.-\sigma_{S 1} \rho_{1.3}\right)+\left(\bar{\sigma}_{r} \sigma_{R 2} \sigma_{2 \mid 1}+\sigma_{S 2} \rho_{2.4 \mid 1}\right)\left(\sigma_{S 2} \rho_{2.4 \mid 1}\right. \\
& \left.-\sigma_{S 1} \rho_{2.3 \mid 1}\right)+\sigma_{S 2} \rho_{3.4 \mid 1.2}\left(\sigma_{S 2} \rho_{3.4 \mid 1.2}-\sigma_{S 1} \sigma_{3 \mid 1.2}\right) \\
& \left.+\left(\sigma_{S 2} \sigma_{4 \mid 1.2 .3}\right)^{2}\right) T+\left(n_{2}+n_{12}\right) \varepsilon_{2}^{2} \\
& \Phi_{7}=\operatorname{cov}\left[\left(-\int_{0}^{T} r_{t} d t\right)\right. \\
& \left.+\ln \left(\frac{S_{T}^{(2)}}{S_{0}^{(2)}}\right), \ln \left(\frac{S_{T}^{(1)} S_{0}^{(2)}}{S_{T}^{(2)} S_{0}^{(1)}}\right)\right]=\left(\sigma _ { S 2 } \rho _ { 1 . 4 } \left(\sigma_{S 1} \rho_{1.3}\right.\right. \\
& \left.-\sigma_{S 2} \rho_{1.4}\right)+\sigma_{S 2} \rho_{2.4 \mid 1}\left(\sigma_{S 1} \rho_{2.3 \mid 1}-\sigma_{S 2} \rho_{2.4 \mid 1}\right) \\
& +\sigma_{S 2} \rho_{3.4 \mid 1.2}\left(\sigma_{S 1} \sigma_{3 \mid 1.2}-\sigma_{S 2} \rho_{3.4 \mid 1.2}\right) \\
& \left.+\left(\sigma_{S 2} \sigma_{4 \mid 1.2 .3}\right)^{2}\right) T+\left(n_{2}+n_{12}\right) \varepsilon_{2}^{2} \\
& \Phi_{8}=\operatorname{cov}\left[\left(-\int_{0}^{T} r_{t} d t\right)\right. \\
& \left.+\ln \left(\frac{S_{T}^{(1)}}{S_{0}^{(1)}}\right), \ln \left(\frac{S_{T}^{(2)} S_{0}^{(1)}}{S_{T}^{(1)} S_{0}^{(2)}}\right)\right]=\left(\sigma _ { S 1 } \rho _ { 1 . 3 } \left(\sigma_{S 2} \rho_{1.4}\right.\right. \\
& \left.-\sigma_{S 1} \rho_{1.3}\right)+\sigma_{S 1} \rho_{2.3 \mid 1}\left(\sigma_{S 2} \rho_{2.4 \mid 1}-\sigma_{S 1} \rho_{2.3 \mid 1}\right) \\
& \left.+\sigma_{S 1} \sigma_{3 \mid 1.2}\left(\sigma_{S 2} \rho_{3.4 \mid 1.2}-\sigma_{S 1} \sigma_{3 \mid 1.2}\right)\right) T+\left(n_{1}+n_{12}\right) \\
& \cdot \varepsilon_{1}^{2} \\
& \Phi_{9}=\operatorname{cov}\left[\left(-\int_{0}^{T} r_{t} d t\right)\right. \\
& \left.+\ln \left(\frac{S_{T}^{(1)} S_{T}^{(2)}}{S_{0}^{(1)} S_{0}^{(2)}}\right), \ln \left(\frac{S_{T}^{(1)} S_{T}^{(2)}}{S_{0}^{(1)} S_{0}^{(2)}}\right)\right] \\
& =\left(\left(\bar{\sigma}_{r}\left(\sigma_{R 1}+\sigma_{R 2} \rho_{1.2}\right)+\sigma_{S 1} \rho_{1.3}+\sigma_{S 2} \rho_{1.4}\right)\right. \\
& \cdot\left(2 \bar{\sigma}_{r}\left(\sigma_{R 1}+\sigma_{R 2} \rho_{1.2}\right)+\sigma_{S 1} \rho_{1.3}+\sigma_{S 2} \rho_{1.4}\right) \\
& +\left(\bar{\sigma}_{r} \sigma_{R 2} \sigma_{2 \mid 1}+\sigma_{S 1} \rho_{2.3 \mid 1}+\sigma_{S 2} \rho_{2.4 \mid 1}\right)\left(2 \bar{\sigma}_{r} \sigma_{R 2} \sigma_{2 \mid 1}\right. \\
& \left.+\sigma_{S 1} \rho_{2.3 \mid 1}+\sigma_{S 2} \rho_{2.4 \mid 1}\right)+\left(\sigma_{S 1} \sigma_{3 \mid 1.2}+\sigma_{S 2} \rho_{3.4 \mid 1.2}\right)^{2} \\
& \left.+\left(\sigma_{S 2} \sigma_{4 \mid 1.2 .3}\right)^{2}\right) T+\left(n_{1}+n_{12}\right) \varepsilon_{1}^{2}+\left(n_{2}+n_{12}\right) \varepsilon_{2}^{2} \text {. }
\end{aligned}
$$

(4) Miscellaneous. One has

$$
\begin{gathered}
\vartheta(a, b)=\ln \left(\frac{\left(\alpha_{a} S_{0}^{(a)}\right)}{\left(\alpha_{b} S_{0}^{(b)}\right)}\right) \\
\bar{\mu}_{r}=E\left[\int_{0}^{T} r_{t} d t\right]
\end{gathered}
$$

$$
\begin{aligned}
= & \frac{r_{0}}{a}\left(1-e^{-a T}\right) \\
& +a \int_{0}^{T}\left(\int_{0}^{t} e^{-a(t-u)} b(u) d u\right) d t \\
\bar{\sigma}_{r}= & \frac{1}{a \sqrt{T}} \sqrt{T-\frac{2}{a}\left(1-e^{-a T}\right)+\frac{1}{2 a}\left(1-e^{-2 a T}\right)} .
\end{aligned}
$$

\section{Competing Interests}

The author declares that there are no competing interests regarding the publication of this paper.

\section{References}

[1] M. Bouzoubaa and A. Osseiran, Exotic Options and Hybrids, John Wiley \& Sons, New York, NY, USA, 2010.

[2] F. Black and M. Scholes, "The pricing of options and corporate liabilities," Journal of Political Economy, vol. 81, no. 3, pp. 637654, 1973.

[3] T. W. Epps, Pricing Derivative Securities, World Scientific, 2000.

[4] O. Vasicek, "An equilibrium characterization of the term structure," Journal of Financial Economics, vol. 5, no. 2, pp. 177-188, 1977.

[5] D. Brigo and F. Mercurio, Interest Rate Models-Theory and Practice, Springer, Berline, Germany, 2nd edition, 2006.

[6] R. C. Merton, "Option pricing when underlying stock returns are discontinuous," The Journal of Financial Economics, vol. 3, no. 1-2, pp. 125-144, 1976.

[7] J. M. Harrison and D. M. Kreps, "Martingales and arbitrage in multiperiod securities markets," Journal of Economic Theory, vol. 20, no. 3, pp. 381-408, 1979.

[8] J. M. Harrison and S. R. Pliska, "Martingales and stochastic integrals in the theory of continuous trading," Stochastic Processes and Their Applications, vol. 11, no. 3, pp. 215-260, 1981.

[9] R. Stulz, "Options on the minimum or the maximum of two risky assets. Analysis and applications," Journal of Financial Economics, vol. 10, no. 2, pp. 161-185, 1982.

[10] W. Margrabe, "The value of an option to exchange one asset for another," The Journal of Finance, vol. 33, no. 1, pp. 177-186, 1978.

[11] P. Zhang, Exotic Options: A Guide to Second Generation Options, World Scientific, River Edge, NJ, USA, 1998.

[12] H. Geman, N. El Karoui, and J.-C. Rochet, "Changes of numeraire, changes of probability measure and option pricing," Journal of Applied Probability, vol. 32, no. 2, pp. 443-458, 1995.

[13] Z. Drezner and G. O. Wesolowsky, "On the computation of the bivariate normal integral," Journal of Statistical Computation and Simulation, vol. 35, no. 1-2, pp. 101-107, 1990.

[14] A. Genz, "Numerical computation of rectangular bivariate and trivariate normal and $t$ probabilities," Statistics and Computing, vol. 14, no. 3, pp. 251-260, 2004.

[15] R. S. Liptser and A. N. Shiryayev, Theory of Martingales, vol. 49 of Mathematics and Its Applications, Kluwer Academic Publishers, 1989. 


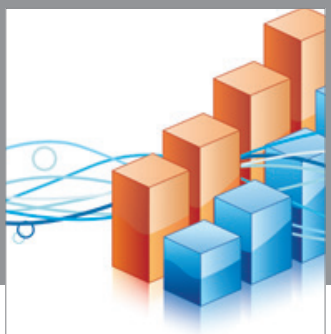

Advances in

Operations Research

vatem alat4

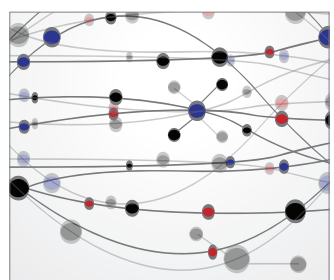

\section{The Scientific} World Journal
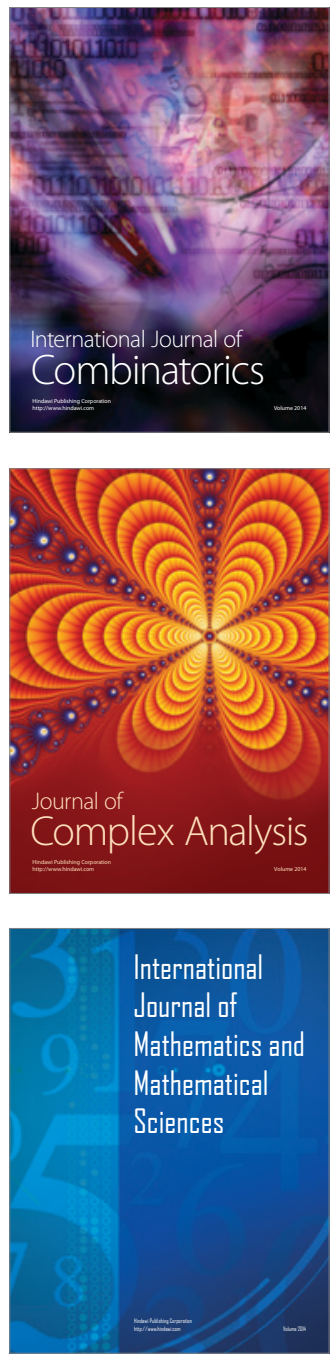
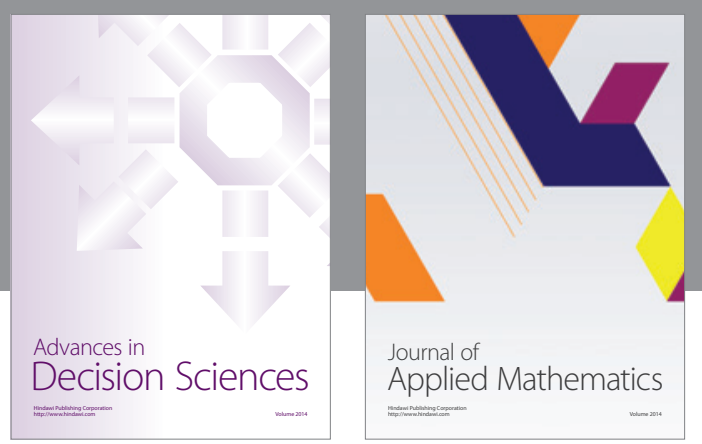

Algebra

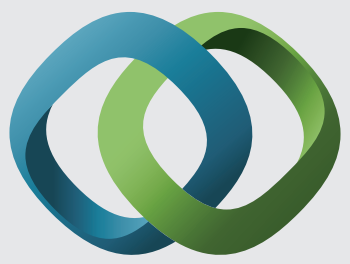

\section{Hindawi}

Submit your manuscripts at

http://www.hindawi.com
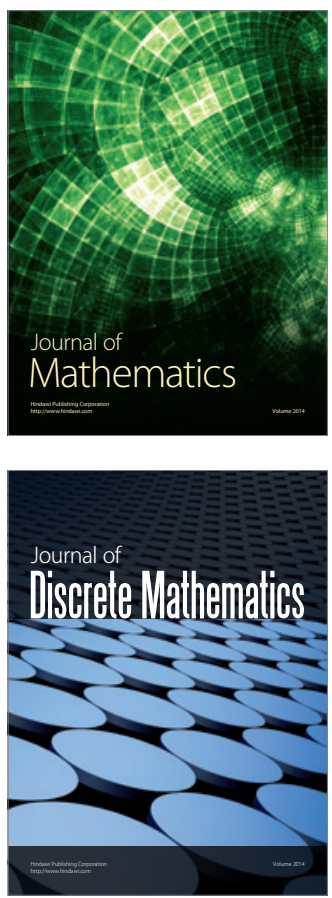

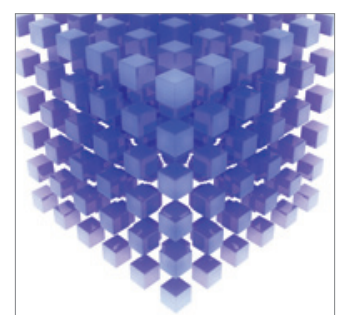

Mathematical Problems in Engineering
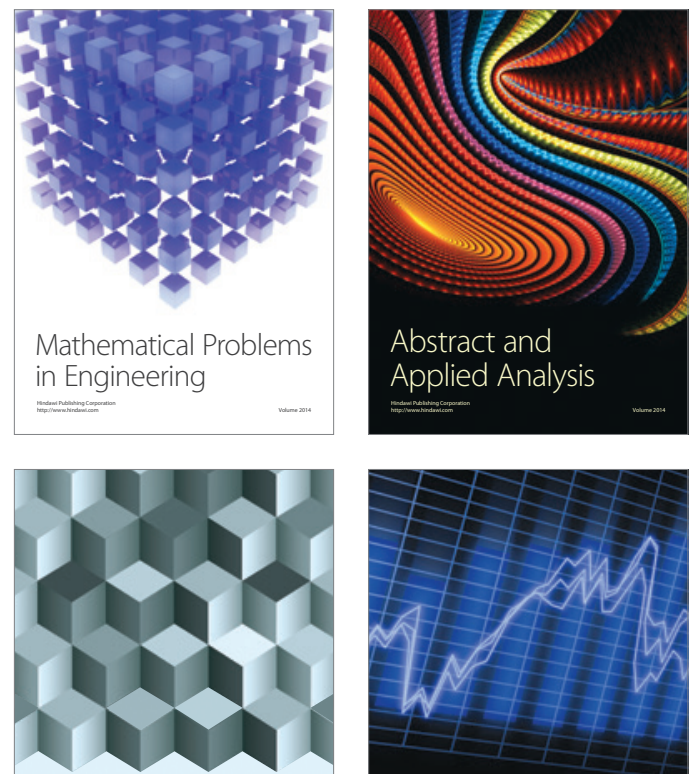

Journal of

Function Spaces

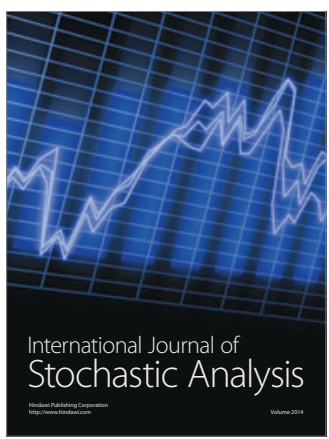

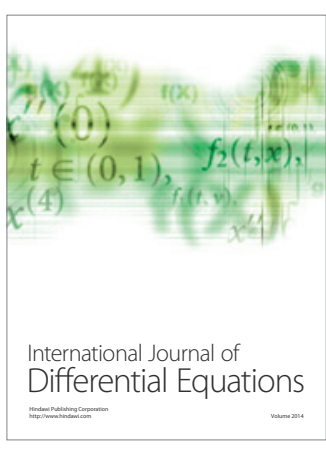
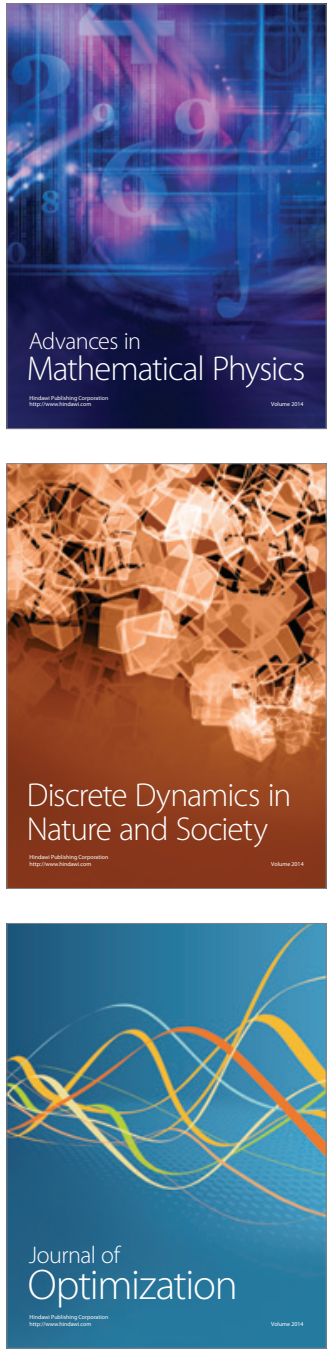